OPEN ACCESS

Edited by:

Yong Teng,

Emory University, United States

Reviewed by:

Qiancheng Zhao,

Yale University, United States

Daniele Vergara,

University of Salento, Italy

*Correspondence:

Youyou Xie

xieyy@enzemed.com

${ }^{\dagger}$ These authors have contribute equally to this work

Specialty section: This article was submitted to Cell Adhesion and Migration,

a section of the journal Frontiers in Cell and Developmental

Biology

Received: 11 August 2021

Accepted: 22 October 2021

Published: 25 November 2021

Citation:

Zhou S, Wang $X$, Ding J, Yang $H$ and Xie $Y$ (2021) Increased ATG5 Expression Predicts Poor Prognosis and Promotes EMT in Cervical Carcinoma. Front. Cell Dev. Biol. 9:757184. doi: 10.3389/fcell.2021.757184

\section{Increased ATG5 Expression Predicts Poor Prognosis and Promotes EMT in Cervical Carcinoma}

\author{
Suna Zhou ${ }^{1,2 t}$, Xuequan Wang ${ }^{1,2 \dagger}$, Jiapei Ding ${ }^{1,2}$, Haihua Yang ${ }^{1,2}$ and Youyou Xie ${ }^{1,2 *}$ \\ ${ }^{1}$ Laboratory of Cellular and Molecular Radiation Oncology, The Affiliated Taizhou Hospital, Wenzhou Medical University, Taizhou, \\ China, ${ }^{2}$ Department of Radiation Oncology, The Affiliated Taizhou Hospital, Wenzhou Medical University, Taizhou, China
}

Cervical cancer has the second-highest incidence and mortality of female malignancy. The major causes of mortality in patients with cervical cancer are invasion and metastasis. The epithelial-mesenchymal transition (EMT) process plays a major role in the acquisition of metastatic potential and motility. Autophagy-related genes (ARGs) are implicated in the EMT process, and autophagy exerts a dual function in EMT management at different phases of tumor progression. However, the role of specific ARGs during the EMT process has not yet been reported in cervical cancer. Based on the data from the Cancer Genome Atlas (TCGA) cervical squamous cell carcinoma and endocervical adenocarcinoma (CESC) sequencing database, we performed the prognosis analysis for those ARGs obtained from the Human Autophagy database. ATG5 was identified as the only important harmful marker influencing survival of cervical cancer patients by univariate Cox regression (HR 1.7; 95\% Cl: $1.0-2.8, p=0.047$ ), and the 5-years survival rate for the high- and low-ATG5 expression groups was $0.486(0.375-0.631)$ and $0.782(0.708-0.863)$, respectively. TCGA CESC methylation data showed that eight methylation sites of ATG5 could also be significantly associated with the overall survival (OS) of cervical cancer patients. Singlesample gene-set enrichment and gene functional enrichment results showed that ATG5 was correlated with some cancer-related pathways, such as phagocytosis-related genes, endocytosis-related genes, immune-related genes, EMT score, and some EMT signaturerelated genes. Next, cell migration and invasion assay and Western blot were applied to detect the function of ATG5 in EMT of cervical cancer. In cervical cancer cells, ATG5 knockdown resulted in attenuation of migration and invasion. The functional study showed that knockdown of ATG5 could reverse EMT process by P-ERK, P-NFkBp65, P-mTOR pathways, and so on. In conclusion, the present study implies that ATG5 was a major contributor to EMT regulation and poor prognosis in cervical cancer.

Keywords: autophagy-related genes, Atg5, autophagy, EMT, cervical cancer, prognosis

\section{INTRODUCTION}

Cervical cancer secondary to breast cancer has the second-highest incidence and mortality of cancer in the female with over 604,127 new cases and 341,831 deaths worldwide per year (Sung et al., 2021). Cervical squamous cell carcinoma and endocervical adenocarcinoma (CESC) are the main pathological types of cervical cancer. Squamous cell carcinomas account for about $75 \%$ of invasive cervical carcinoma cases. The main treatment methods for cervical cancer patients 
include surgery or concurrent radiotherapy and chemotherapy regimens, such as chemotherapy concurrent with radiotherapy and afterloading brachytherapy (Small et al., 2017). Clinical trials including the adoptive T-cell therapy, checkpoint inhibitors, and human papillomavirus (HPV) vaccines have shown satisfactory results. Based on these treatments, 3-years local control rate of patients with early-stage CESC is above $87 \%$. The 5 -years survival rate is $16.5 \%$, and the median survival time is only $8-13$ months for metastatic cervical cancer because of no standard treatment due to their heterogeneous manifestations (van Meir et al., 2014).

In most cases, the patient has already advanced to the moderate or late stages when first diagnosed. Therefore, invasion and metastasis are the major characteristics and causes of mortality in patients with advanced cervical cancer. Cancer cells acquire metastatic potential and gain motility mostly through the epithelial-mesenchymal transition (EMT) process. EMT is a cellular process characterized by the transformation from an epithelial phenotype to a mesenchymal phenotype (Nieto et al., 2016). This process plays a significant role in cancer initiation, invasion, metastasis, tumor immunosuppression, and immune evasion (Nieto et al., 2016; Jiang and Zhan, 2020). Targets associated with EMT regulation preventing cancer invasion and metastasis could be found to improve the prognosis of cervical carcinoma patients.

Novel increasing evidence showed that the process of EMT could be promoted or inhibited by autophagy (Catalano et al., 2015; Dash et al., 2018; Daskalaki et al., 2018; Wang et al., 2019; Wu et al., 2019; Liu et al., 2020). Autophagy is a catabolic procedure involved in maintaining homeostasis by metabolizing intracellular proteins and organelles under various conditions of cellular stress (Dikic and Elazar, 2018). There was a complicated regulatory network between the regulation of EMT and autophagy. EMT-involved oncogenic signal proteins such as SNAI1, SLUG, ZEB1/2, and NOTCH1 were functionally related to autophagy (Zada et al., 2021). Autophagy plays a tumorinhibitory effect in the initial stages of carcinogenesis and a tumor-promotion function during the cancer progression (White, 2012). By its controversial role in tumor, the acquisition of EMT phenotype required autophagy activation during cancer progression and could be inhibited by autophagy inducers in the early stage of tumor formation (Hu et al., 2018; Wang et al., 2020). ARGs, especially the core mammalian autophagy proteins including ATG2-10, 12-14, 16-18, were a series of functional proteins implicated in the regulation of autophagy. However, the role of specific ARGs during the EMT process of cervical cancer has not yet been reported. DNA methylation is an epigenetic modification process that affects the expression of many genes, contributing to genome stability and the regulation of gene transcription (Moore et al., 2013). Methylation genes such as cadherin 1 (CDH1), death-associated protein kinase 1 (DAPK1), telomerase reverse transcriptase (TERT), and cell adhesion molecule 1 (CADM1) have been found as the highest methylation frequency genes (Wentzensen et al., 2009) and identified as promising markers for prognostic prediction of cervical cancer patients (Overmeer et al., 2011; Abudukadeer et al., 2012; Sun et al., 2015). Although some
ARGs have been identified to prospectively predict the prognosis in patients with squamous cell cervical cancer (Chen et al., 2020; Shi et al., 2020), the DNA methylation of ARGs has not been analyzed in the prediction of cervical cancer prognosis.

In this paper, we aimed to find the clinical prognostic values of essential ARGs in cervical cancer based on the TCGA database. We found that a high level of ATG5 expression was an important poor prognostic factor in cervical cancer. In addition, further studies were performed to verify the role and the potential mechanisms of ATG5 in promoting migration and invasion in cervical cancer cell lines. Our results suggested that ATG5 acted as a poor prognostic marker and may be applied as a potential target for reversing the invasion and metastasis in cervical cancer.

\section{MATERIALS AND METHODS}

\section{Data Source and Preprocessing}

RNA-seq reads data, methylation $450 \mathrm{k}$ data, and clinical data of patients with cervical carcinoma from TCGA were acquired from Broad Institute Firehose (https://xenabrowser.net/ datapages/). ARGs were obtained from the Human Autophagy database (HADb http://www.autophagy.lu/project. html). Samples without clinical survival status follow-up data were excluded from the study. Overall, a total of 296 CESC samples were enrolled in the present study. The 31 core ARGs involved in the further analysis are described in Table 1. These ARGs, identified as important regulators of autophagy, were essential for autophagosome formation and were involved in the further result analysis. Rstudio and R programming(4.03) were the principal tools for analyzing data throughout the study.

\section{Survival Analysis of ARGs in CESC}

The optimal cutoff values of ARGs based on the expression Fragments Per kilobase per Million mapped reads (FPKM) value or methylation Beta-value, the survival time, and the survival status were identified by the "surv_cutpoint" function in "survminer" package as described before (Wang et al., 2021). The cutoff value of the OS significant genes is listed in Supplementary Figure S1. The patients with relative values above or below the optimal cutoff were considered as high or low groups for each gene expression level, methylation level, or EMT score. Kaplan-Meier (KM) survival analysis with log-rank test was then used for investigating the OS difference between the abovementioned high and low groups. The correlation between the OS characteristics of CESC patients and each ARG expression was explored by a univariate Cox regression analysis. A multivariate Cox regression analysis was performed to investigate whether each ARG is an independent OS influence factor.

The patients with a relative value above or below the optimal cutoff were considered as high or low group for each gene expression level, methylation level, or EMT score. KM survival analysis with log-rank test was then used for investigating the 
TABLE 1 | Autophagy-related genes and their function in autophagy.

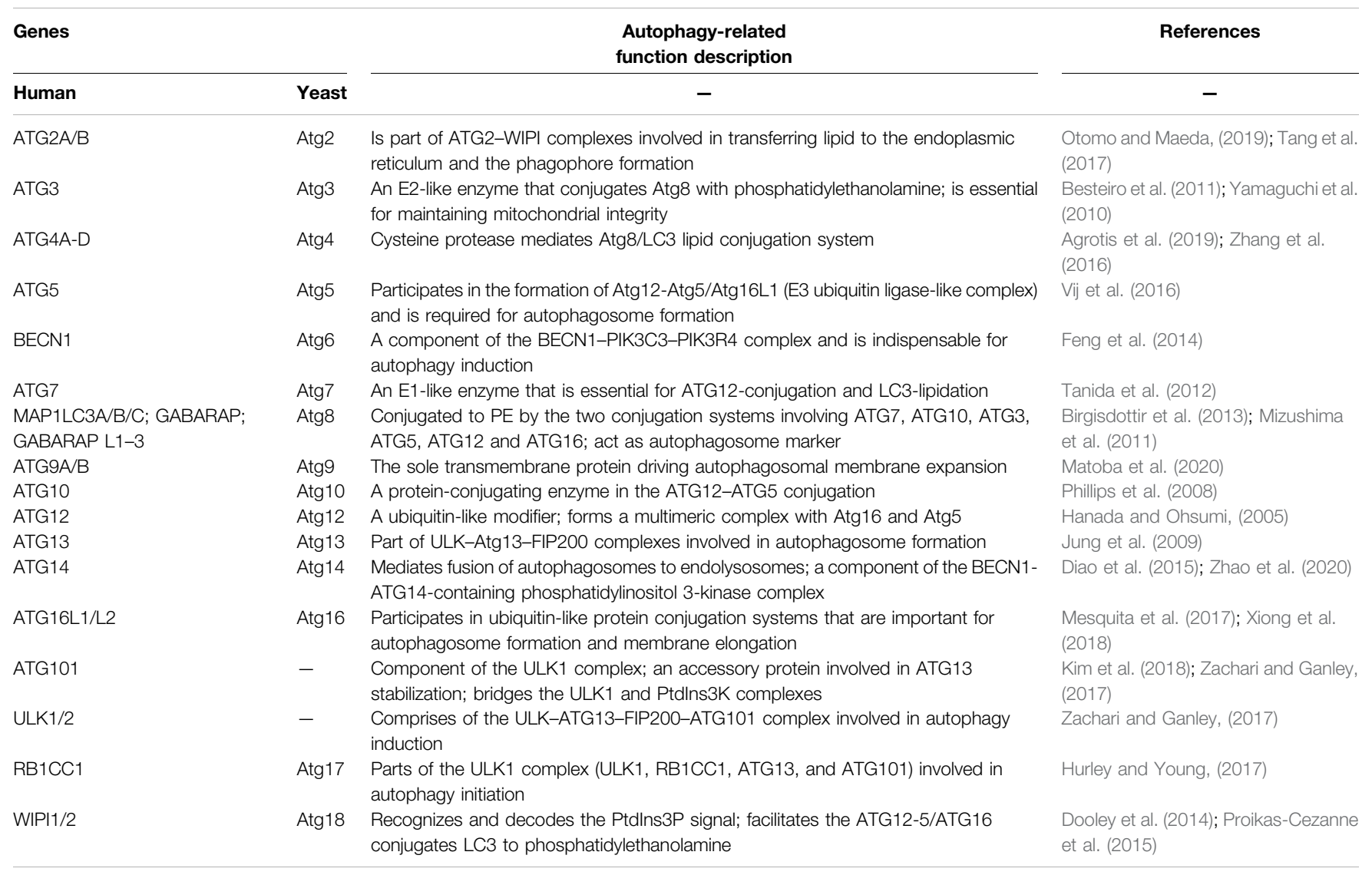

overall survival (OS) difference between the abovementioned high and low groups.

\section{EMT Score Evaluation}

By "GSVA" R package, the EMT score for evaluating the EMT status of each CESC patient was quantified by single-sample gene-set enrichment analysis (ssGSEA) based on previously reported EMT signature genes (Choi et al., 2010; Hanzelmann et al., 2013). The correlation between ATG5 and EMT score or between ATG5 and EMT signature related genes is calculated by Pearson's correlation coefficient and plotted in heatmap by the "circlize" R package. The EMT score density between normal and tumor tissues and the survival difference between high and low groups were also analyzed.

\section{ATG5 Co-Expression Network Selection and Gene Functional Enrichment Analysis}

The genes co-expressed with ATG5 in CESC were screened according to the Pearson correlation coefficient ( $\mid$ cor $\mid>0.3$, $p<0.05)$ and plotted in the volcano plot by the "ggplot2" package. Heatmaps of the top 50 negative or the top 50 positive ATG5 expression correlated genes were plotted by the "pheatmap" R package. Metascape (Zhou et al., 2019) was employed to gain insights into the biological functions of those co-expressed genes of ATG5.

\section{Identification Prognostic Methylation Sites of ATG5}

The methylation site information of ATG5 in TCGA CESC methylation data based on Illumina Infinium Human Methylation450 BeadChip platform was mapped by an annotation file retrieved from the hg19 GPL16304 legacy annotation file (Price et al., 2013). The methylation level of each site in ATG5 was grouped into low and high by the survminer package as mentioned above. The OS significant associated sites were screened through KM survival analysis. Besides, MEXPRESS was used for visualizing expression, DNA methylation, and clinical parameters based on TCGA data (http://mexpress.be). The correlation between the methylation level of each site of ATG5 and the expression of ATG5 is calculated by the "corrplot" package (Wei and Simko, 2021).

\section{Gene Mutation Analysis of ARGs in CESC}

Waterfall plots of ARGs mutation were produced by the oncoplot function in the "maftools" R package (Mayakonda et al., 2018) to visualize the mutation status such as classification and frequency of mutation types, frequency of variant types, and frequency of SNV classes for CESC samples. The mutational exclusion and cooccurrence mutations among those ARGs were analyzed by Fisher's exact test (Mayakonda et al., 2018). The variant allele frequency (VAF) distribution of ARGs was also plotted. 


\section{Validation of the Prognosis Performance for Different Clinical Features of CESC}

ALL CESC patients were grouped based on different clinical staging information (pathological-T/N/M, histologic-Grade/ Stage) or the age at diagnosis ( $<45$ years, $45-69$ years, and $>69$ years). Subtypes of CESC were grouped into squamous cell neoplasms or adenocarcinoma based on the clinical data. The OS difference between the high- or low-ATG5 expression groups was evaluated in KM survival analysis.

\section{Cell Lines}

Hela, Ca Ski (human cervical cancer cell lines), and PANC-1 cells (pancreatic cancer cell line) were purchased from the American Type Culture Collection (ATCC, Manassas, VA, United States). Hela cells were incubated in DMEM supplemented with $10 \%$ FBS (Gibco, Grand Island, NY). Ca Ski and PANC-1 cells were incubated in RPMI 1640 containing 10\% FBS (Gibco, Grand Island, NY). PANC-1 cells were used as a positive control for ATG5 expression (Yang et al., 2015). Cells are cultured in an incubator at $37^{\circ} \mathrm{C}$ with $5 \% \mathrm{CO}_{2}$.

\section{Cell Transfection}

According to the manufacturer's instructions (GeneChem Corporation, Shanghai, China), Hela and Ca Ski cells were transfected with the recombinant lentiviral vector LV-ATG5RNAi labeled with green fluorescent to establish ATG5knockdown cells and named as KD. In brief, the cells were digested at the logarithmic growth phase, then resuspended in the complete medium at a concentration of $3-5 \times 10^{4} \mathrm{cells} / \mathrm{ml}$, and seeded in six-well plates. At $20 \%$ confluence, cells were transfected with the recombinant lentivirus for $16 \mathrm{~h}$. Subsequently, a conditioned medium containing transfection reagent was refreshed. At $72 \mathrm{~h}$ post-infection, the positive expression rate of GFP was used to evaluate the transfection efficiency under a fluorescence microscope (Olympus Corporation, Tokyo, Japan). siRNAs specific for ATG5 were obtained from GenePharma (Shanghai, China), and the detailed sequences were as follows: ATG5-RNAi (98917-1) sense: CCTTTCATTCAGAAGCT GTTT, ATG5-RNAi (98918-1) sense: CCTGAACAGAA TCATCCTTAA, ATG5RNAi (98919-1) sense: GATTCATGGAATTGAGCCAAT, NC-RNAi sense: TTCTCCGAACGTGTCACGT.

\section{Quantitative Real-Time Polymerase Chain Reaction (qRT-PCR)}

Cells were collected and lysed by Trizol reagent (Invitrogen, Carlsbad, CA). Next, cDNA was obtained by using M-MLV reverse transcriptase (Promega, Madison, WA). Using a LightCycler 480 II RT-PCR System (Roche, Basel, Switzerland), qRT-PCR was performed as follows: $95^{\circ} \mathrm{C}$ for $30 \mathrm{~s}$, and then 40 cycles of $95^{\circ} \mathrm{C}$ for $5 \mathrm{~s}$ and $60^{\circ} \mathrm{C}$ for $30 \mathrm{~s}$. The sequences of primers amplified in this study were as follows: ATG5: forward 5'-AAGAGTGTTTATTCG TCGGT-3', reverse $5^{\prime}$-ATCACAGCTTAGTGTTCCCT-3' ${ }^{\prime}$. ACTB: forward $5^{\prime}$-GCG TGACATTAAGGAGAAGC-3', reverse $5^{\prime}$-CCACGTCACA
CTTCATGATGG-3'. Beta-actin (ACTB) gene was used as an internal reference.

\section{Western Blot Assay}

In short, cells were harvested and washed with PBS twice, and then lysed in RIPA buffer (Beyotime, Shanghai, China) on ice for $15 \mathrm{~min}$. Then, BCA Protein Assay Kit (Beyotime, Shanghai, China) was used to detect the protein concentration in the supernatant from crushed cells by ultrasonication. The final concentration was adjusted to $2 \mu \mathrm{g} / \mu \mathrm{l}$ by fresh RIPA solution, followed by protein denaturation. $10 \%$ SDS-PAGE was applied to separate the protein samples and then transferred onto PVDF membranes (Millipore, Burlington, MA). After that, membranes were incubated with TBST containing 5\% skim milk to block non-specific antigens. Next, the membranes were incubated with primary antibodies and HRP-conjugated second antibodies in turn. Finally, the protein bands were visualized by the electrochemiluminescence (ECL) detection system. The primary antibodies used include mouse anti-ATG5 antibody (1:500, sc-133158, Santacruz, Santa Cruz, CA), mouse antiERK antibody (1:2,000, \#9107,CST, Danvers, MA, United States), rabbit anti-NFkB p65 antibody (1:3,000, \#8242, CST, Danvers, MA, United States), rabbit anti-mTOR antibody (1:1,000, \#2983, CST, Danvers, MA, United States), rabbit anti- $p$ ERK antibody (1:1,000, \#4376, CST, Danvers, MA, United States), rabbit anti- $p$-NFkB p65 antibody (1:500, ab76302, Abcam, Cambridge, MA, United States), rabbit anti- $p$-mTOR antibody (1:500, ab109268, Abcam, Cambridge, MA, United States), rabbit anti-P38 antibody $(1: 3,000, \# 8690$, CST, Danvers, MA, United States), rabbit anti-AKT antibody (1:500, \#4691, CST, Danvers, MA, United States), mouse anti-E-Cadherin antibody (1:500, \#14472, CST, Danvers, MA, United States), rabbit anti-pP38 antibody (1:5,000, \#4631, CST, Danvers, MA, United States), rabbit anti- $p$-AKT antibody (1:1,000, \#4060, CST, Danvers, MA, United States), rabbit anti-N-Cadherin antibody (1:500, ab18203, Abcam, Cambridge, MA, United States), mouse anti-Twist antibody (1:100, ab50887, Abcam, Cambridge, MA, United States), rabbit anti-c-Myc antibody (1:1,000, ab32072, Abcam, Cambridge, MA, United States), rabbit anti-Snail antibody (1:500, \#3879, CST, Danvers, MA, United States), rabbit anti- $\beta$-Catenin antibody (1:2,000, \#8480, CST, Danvers, MA, United States), rabbit anti-Slug antibody (1:500, \#9585, CST, Danvers, MA, United States), rabbit anti-p- $\beta$-Catenin antibody (1:500, \#2009, CST, Danvers, MA, United States), rabbit antiMMP-2 antibody (1:500, \#40994, CST, Danvers, MA, United States), mouse anti-Fibronectin antibody (1:500, MAB 1918, R\&D Systems, Minneapolis, MN, United States), rabbit anti-Vimentin antibody (1:500, \#5741, CST, Danvers, MA, United States), and rabbit anti-MMP-9 antibody (1:500, \#13667, CST, Danvers, MA, United States). Mouse anti- $\beta$-actin (1:5,000, sc-69879, Santacruz, Santa Cruz, CA) and mouse antiGAPDH (1:10,000, ab37168, Abcam, Cambridge, MA, United States) were used as an internal control.

\section{Cell Migration and Invasion Assays}

Cells were seeded in the upper chamber coated with (invasion) or without (migration) Matrigel (Corning Costar, Cambridge, MA, 

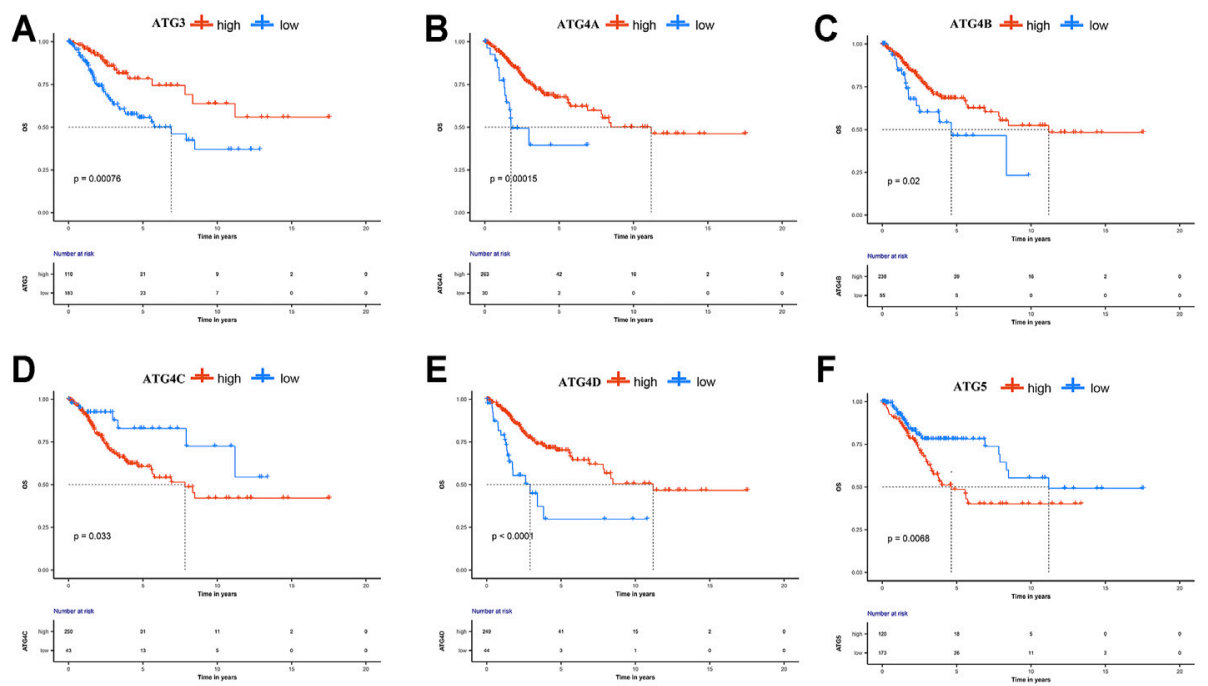

G
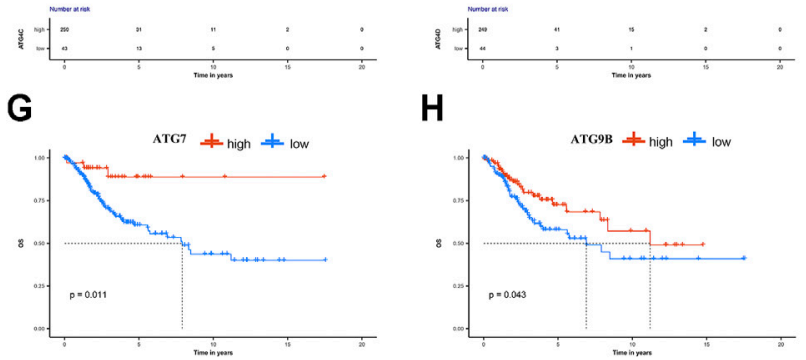

H
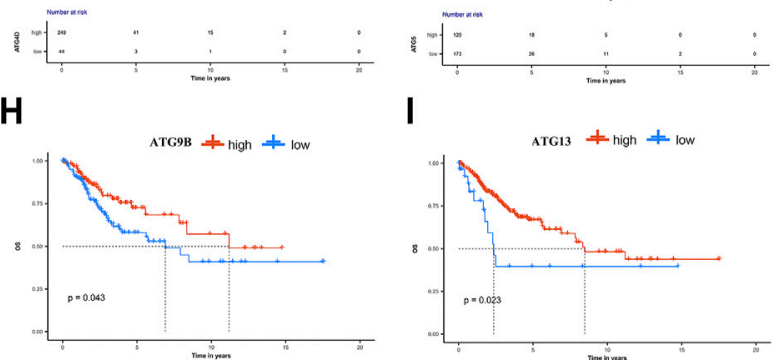

I

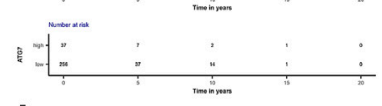

J

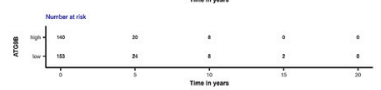

K
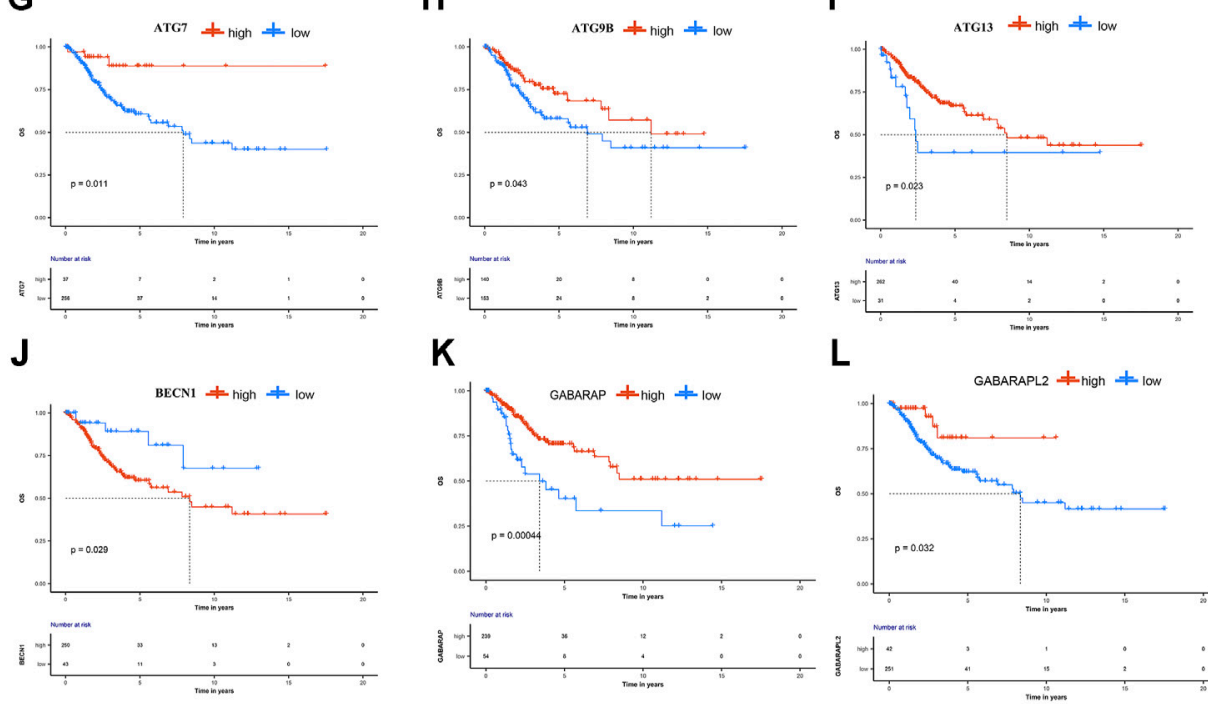

$\mathbf{L}$
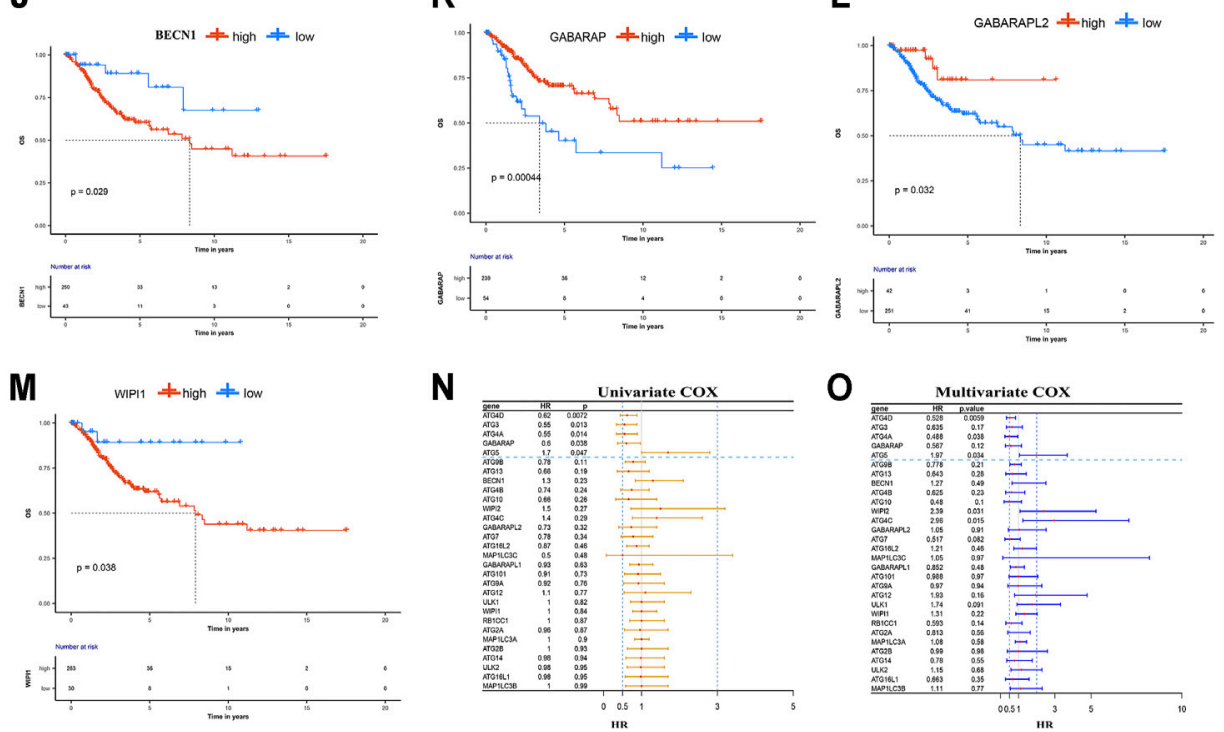

FIGURE 1 | Overall survival analysis on ARGs in cervical cancer. The expression of (A) ATG3 (B) ATG4A (C) ATG4B (E)ATG4D (G) ATG7 (H) ATG9B (I) ATG13 (K) GABARAP, and (L) GABARAPL2 were associated positively with OS, while increased expression of (D) ATG4C (F) ATG5 (J) BECN1, and (M) WIPI1 negatively correlated with OS. Figure (N, O) According to the forest plot for the Univariate and Multivariate Cox regression analysis results, ATG5 was a risk prognostic factor, while ATG4D, GABARAP, ATG4A, and ATG3 were favorable prognostic factors in CESC patients. And, ATG5, ATG4D, and ATG4A were independent prognostic factors.

United States) at a density of $1 \times 10^{5}$ cells/well. In invasion assay, serum-free medium was added into the lower chamber. In the migration assay, a medium containing $30 \%$ FBS was added to the lower chamber. The noninvasive/non-migrated cells were removed by a cotton swab after 48 -h incubation. Then, the cells were fixed in $4 \%$ paraformaldehyde for $0.5 \mathrm{~h}$ and stained with $0.5 \%$ crystal violet solution, followed by taking photographs through the microscope. 

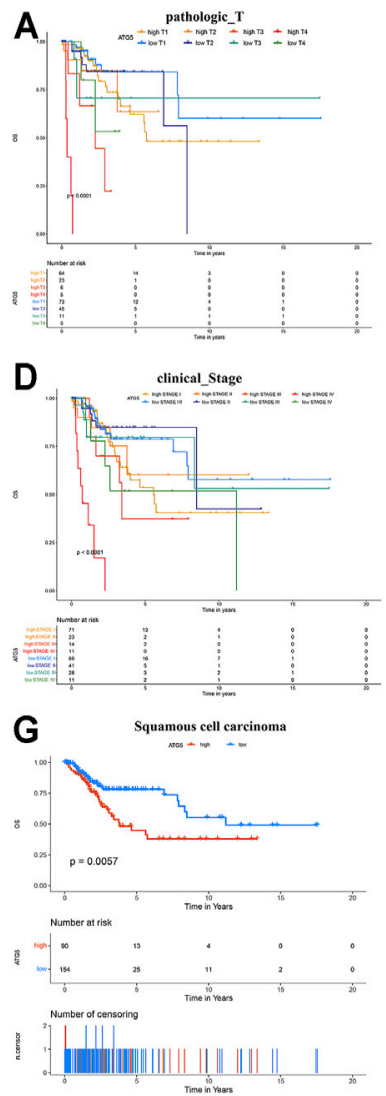
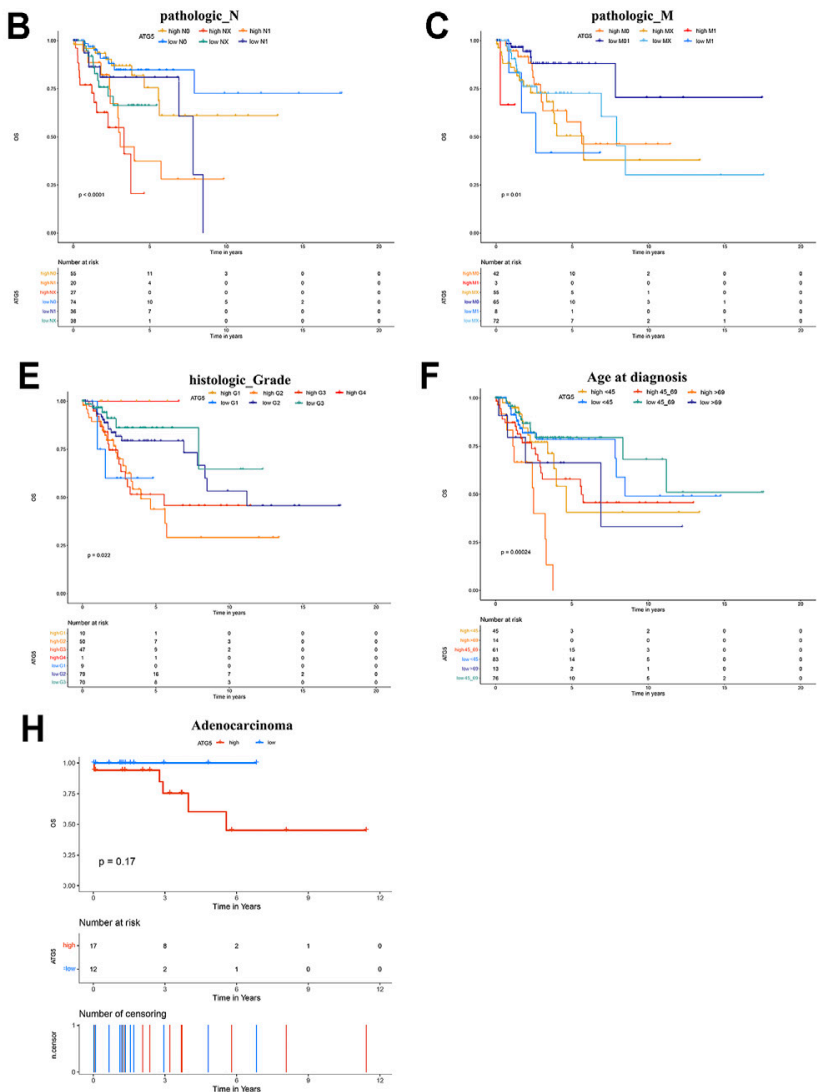

FIGURE 2 | Kaplan-Meier survival analysis of ATG5 compared with different clinical parameters. By comparison, high expression of ATG5 in cervical patients with most different (A) T stage (B) N stage (C) M stage (D) FIGO stage (E) pathological grade, and (F) Ages at diagnosis showed shorter median OS time; Cervical patients with (G) Cervical squamous cell carcinoma, and (H) Cervical adenocarcinoma had shorter OS in ATG5 overexpression group.

\section{Statistical Analysis}

All molecular biology assays were repeated in triplicate. Experimental data are presented as the mean \pm SD. R (version 4.03) and Rstudio software were applied to perform statistical analysis. The differences between the groups were analyzed via Student's $t$-test and Mann-Whitney $U$ test. Univariate and multivariate Cox regression analysis were performed to identify the factors influencing prognosis. Survival analysis was done using the KM method and the log-rank test. $p<0.05$ was designated as statistically significant.

\section{RESULTS}

\section{Baseline Information of the CESC Patients}

A total of 309 sequencing data and 312 methylation data in TCGA CESC cohort were obtained from the TCGA data. The median age of those 306 participants was 46.57 years (min 20.95 years and max 89.04 years). The median follow-up time for CESC patients with survival information was 1.87 years (range $0-17.55$ years), and among them, 225 were alive and 74 were dead during the follow-up time.

\section{Prognosis of ARGs in CESC}

High expression levels of ATG4C, ATG5, BECN1, and WIPI1 were associated with poor prognosis in CESC, while CESC patients with overexpression of ATG3, ATG4A, ATG4B, ATG4D, ATG7, ATG9B, ATG13, GABARAP, and GABARAPL2 have longer OS and higher 5-years survival rate (Figure 1). Furthermore, the univariate Cox regression results indicated that ATG5, ATG4D, GABARAP, ATG4A, and ATG3 were significantly associated with OS (Figure 1N). ATG5 was identified as a risk prognostic predictor in CESC patients (HR 1.7; 95\% CI, 1.0-2.8, $p=0.047$ ), while another four ARGs were identified as favorable prognostic factors ( $\mathrm{HR}<1, p<0.05$ ). Subsequently, the multivariate Cox results showed that ATG5, ATG4D, and ATG4A were independent prognostic factors affecting OS of patients with CESC (Figure 10). Among them, high expression of ATG5 or low expressions of ATG4D and ATG4A were associated with unfavorable prognosis. These results indicated that ATG5 among these ARGs was the most harmful factor affecting the prognosis of CESC patients. According to the $\mathrm{KM}$ analysis, the 5-years survival rate for high- and low-expression groups of ATG5 is 0.486 $(0.375-0.631)$ and $0.782 \quad(0.708-0.863)$ respectively. 

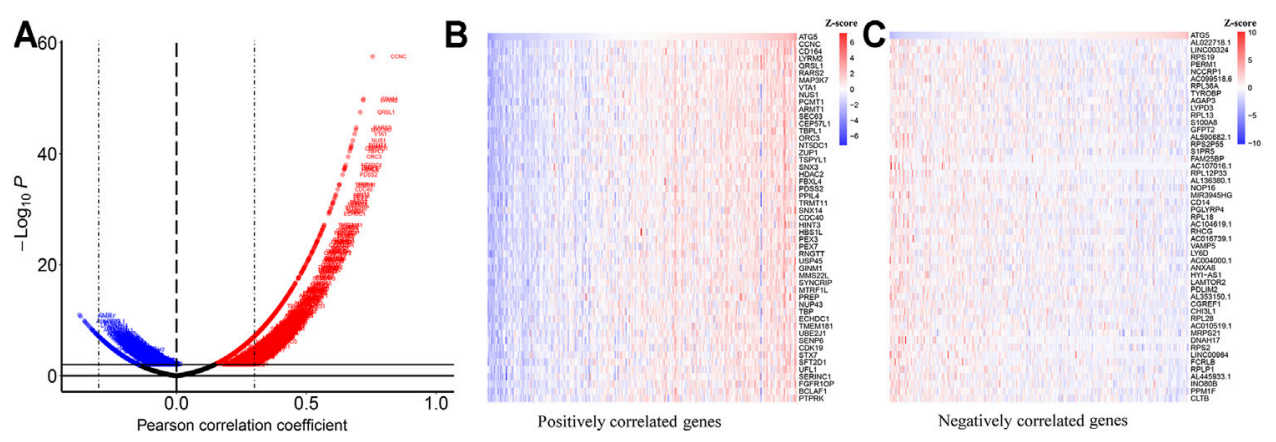

FIGURE 3 | Co-expression genes correlated with ATG5 in cervical cancer (LinkedOmics) (A) Pearson test was applied to analyze the correlation between ATG5 and differentially expressed genes in Cervical cancer. Blue dots $(R<-0.3, p<0.01)$ represent as negatively correlated genes, while red dots $(R>0.3, p<0.01)$ represent as positively correlated genes (B) Heat map showed the top 50 significant genes positively correlated with ATG5 (C) Heat map showed the top 50 significant genes negatively correlated with ATG5 (The expression of each in each heatmap is normalized with Z-score).

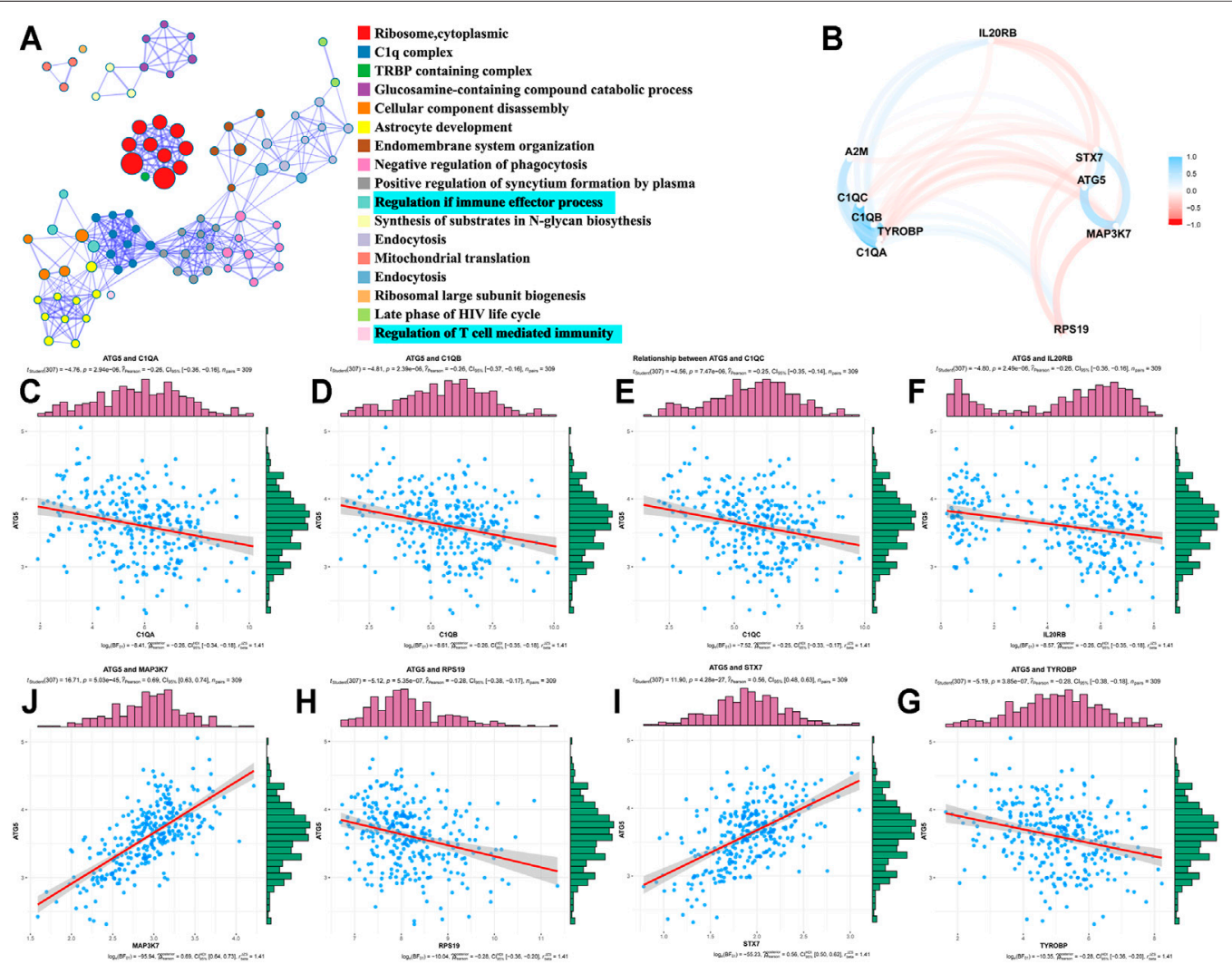

FIGURE 4 | GO analysis and correlation analysis (A) GO analysis revealed the biological processes and molecular functions involved in the top 100 ATG5-related co-expressed genes by "metascape" data (B) The correlation between ATG5 and co-expressed genes involved in the regulation of the immune effector process in cervical cancer patients. Blue lines represent as negatively correlated genes, while red lines represent as positively correlated genes (C-J) Among those regulation of the immune effector process genes, ATG5 expression was significantly negatively correlated with MAP3K7, STX7 and significantly positively related to A2M, C1QA, C1QB, C1QC, RPS19, TYROBP, and IL20RB.

Furthermore, KM survival analysis was also performed to show that ATG5 expression could effectively predict the prognosis of patients with CESC with different clinical stages, pathological grades, and ages at diagnosis (Figures $\mathbf{2 A - F}$ ). For patients with cervical squamous cell carcinoma, patients with ATG5 overexpression have significantly shorter OS than the ATG5 down-expression group (Figure 2G). For cervical adenocarcinoma, due to the limited sample size of patients included in the analysis, the $p$-value of the $\mathrm{km}$ survival curve is not significant, but patients with ATG5 down-expression 

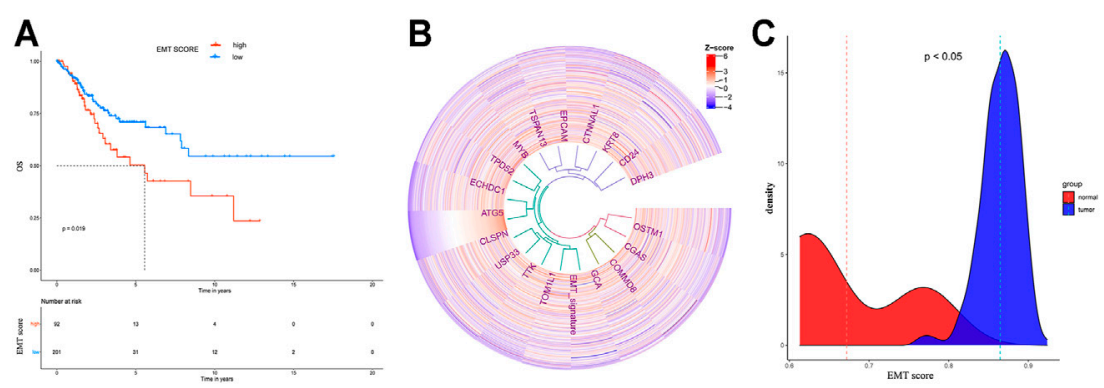

FIGURE 5 | The correlation between ATG5 and co-expressed genes involved in the regulation of EMT in cervical cancer patients. A. the Kaplan-Meier survival plot showed that the median OS time of low EMT score patients was significant longer than high EMT score patients. B. ATG5 showed co-expressed ship with genes involved in the regulation of EMT in cervical cancer patients. C. The EMT signature showed significant difference in cervical normal and cancer tissues.

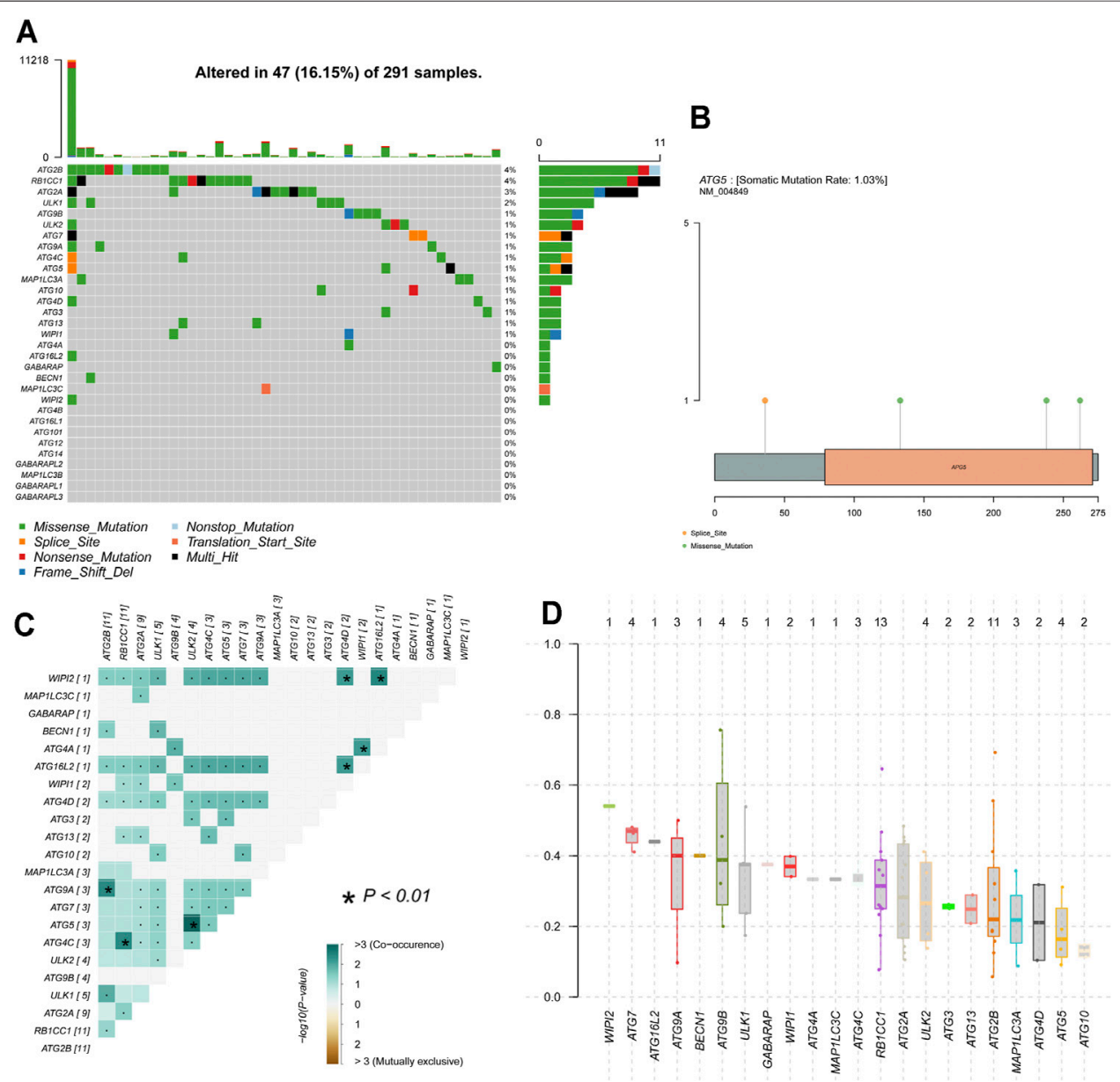

FIGURE 6 | Mutation data analysis of ARGs in CESC (A) Waterfall plots of ARGs mutation results show that ATG2B and RB1CC1 are the most frequently mutated genes. A total of 16 genes has a mutation rate $\geq 1 \%$ (B) mutation type and domain of ATG5 (C) The co-occurrence of ARGs mutation in cervical cancer. Dot represents as no statistical significance, ${ }^{\star} p<0.01$. Green means co-occurrence (D) The variant allele frequency (VAF) distribution of ARGs in CESC by plotvaf function in "maftools" package. Each dot in the boxplot represents a variant. The total number of variants is list on the top of each box. 

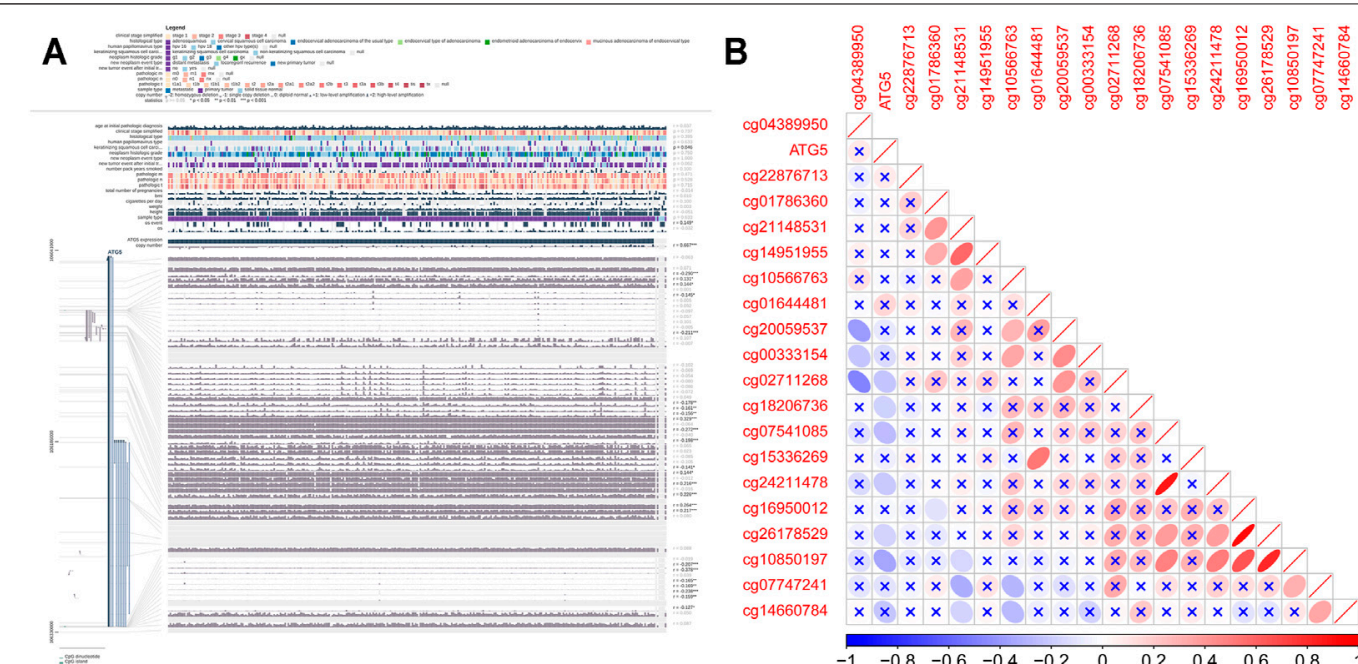

$\operatorname{cg} 04389950, /$
ATG5

cg22876713 $\times \times /$

cg01786360 $\times \times \times$

$\operatorname{cg} 21148531 \times \times \times$

$\operatorname{cg} 14951955 \times \times \times 10$

$\operatorname{cg} 10566763 \times \times \times \times \times \times$

$\operatorname{cg} 01644481 \times \times \times \times \times \times \times$

$\operatorname{cg} 20059537 \times \times \times \times \times \times \times$

cg00333154 $\times \times \times \times \times \times$

cg02711268 $\times \times \times \times \times \times \times /$

cg18206736 $\times$ × $\times \times \times \times \times \times \times \times /$

cg07541085 $\times$ X $\times \times \times \times \times \times \times \times \times$

cg15336269 $\times \times \times \times \times \times \times \times \times \times \times \times$

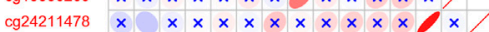

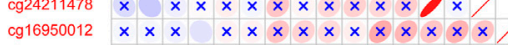

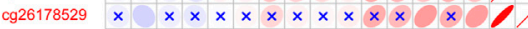

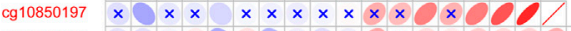

$\operatorname{cg} 07747241 \times \times \times \times \times \times \times \times \times \times \times \times \times \times \times$

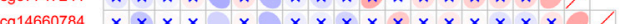

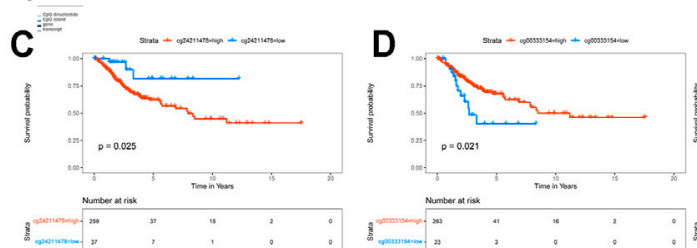

$\begin{array}{llllllllllll}-1 & -0.8 & -0.6 & -0.4 & -0.2 & 0 & 0.2 & 0.4 & 0.6 & 0.8 & 1\end{array}$

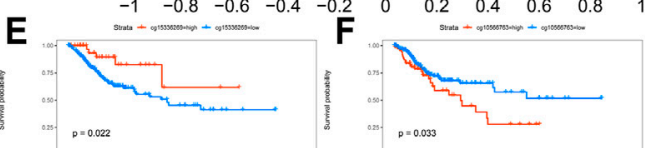

$p=0.022$

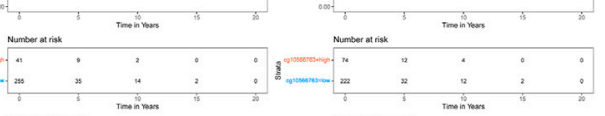

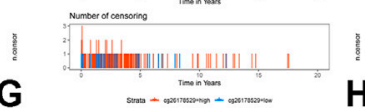
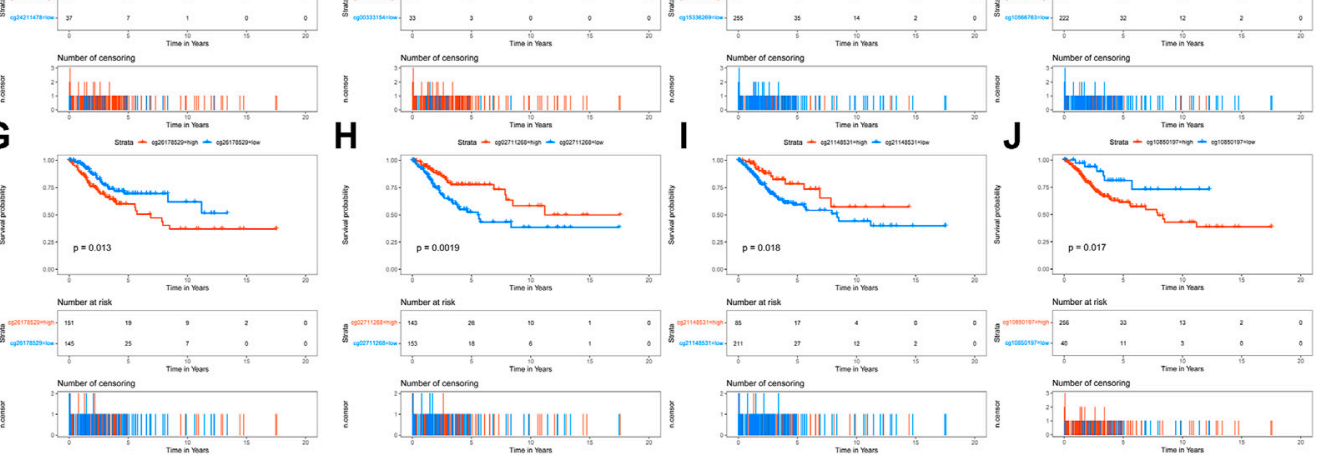

FIGURE 7 | Genomic methylation of ARGs in CESC (A) The methylation of ATG5 gene was significantly correlated with the OS in cervical cancer patients (B) The correlation between methylation sites of ATG5 and ATG5 expression in cervical cancer patients. Purple represents negative correlation; red represents positive correlation; " $\times$ " represents no statistical significance (C-J) Kaplan-Meier survival analysis showed the methylation sites of ATG5 significantly related to OS in cervical cancer patients ( $p$ 0.05). cg00333154, cg15336269, cg02711268, and cg21148531 located in ATG5 were beneficial for OS, while cg24211478, cg10566763, cg26178529, and cg10850197 located in ATG5 were harmful for OS.

showed a trend towards a longer survival with no death during the follow-up times (Figure $\mathbf{2 H}$ ).

\section{Co-Expression Genes Correlated With ATG5 in CESC}

Based on the TCGA database, we analyzed the co-expressed genes of ATG5 in 304 CESC patients through the Pearson correlation coefficient. As shown in Figure 3A, red dots represented 5,666 genes positively associated with ATG5, while blue dots represented 2,114 genes negatively associated with ATG5. The heat map was used to show the top 50 genes positively or negatively correlating with ATG5 (Figures 3B,C). Next, GO enrichment analysis was performed to investigate the biological pathway of the top ATG5-related co-expressed genes. ATG5 co-expressed genes are correlated with ribosome, cytoplasmic, C1 complex, TRBP-containing complex, glucosamine-containing compound catabolic process, and so on. There are concerns that ATG5 also participated in the regulation of the immune effector process and regulation of T-cell-mediated immunity (Figure 4A). We further found that the ATG5 co-expressed genes involved in the regulation of the immune effector process were A2M, C1QA, C1QB, C1QC, RPS19, MAP3K7, TYROBP, STX7, and IL20RB. MAP3K7, STX7, and IL20RB were involved in the regulation of T-cellmediated immunity. Moreover, ATG5 expression was inversely associated with MAP3K7 and STX7 and positively related to A2M, C1QA, C1QB, C1QC, RPS19, TYROBP, and IL20RB (Figures 4B-J). Furthermore, it was important to investigate the relation between ATG5 and EMT-related gene signature. The result is represented in Figure 5. We found that ATG5 was significantly correlated with EMT scores quantified by ssGSEA 


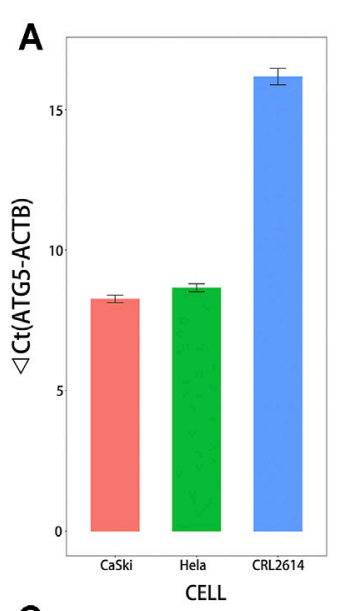

C

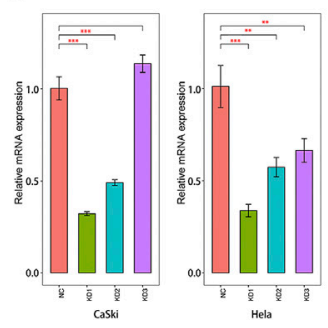

B

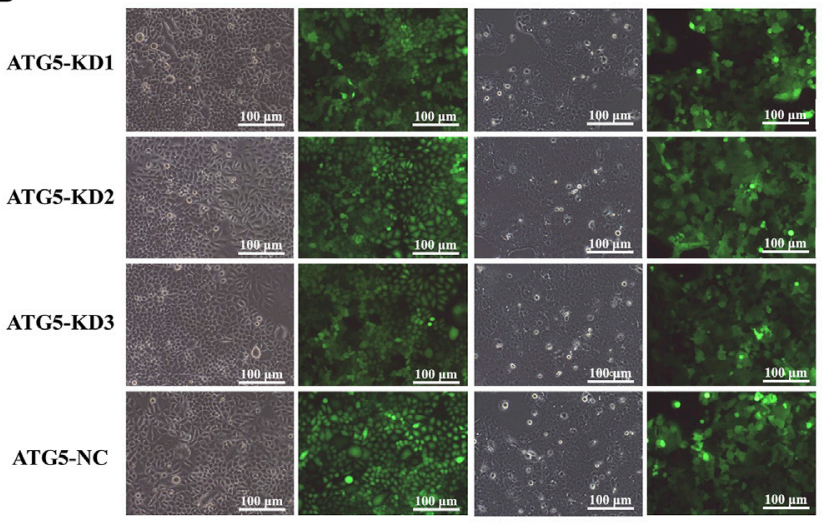

D

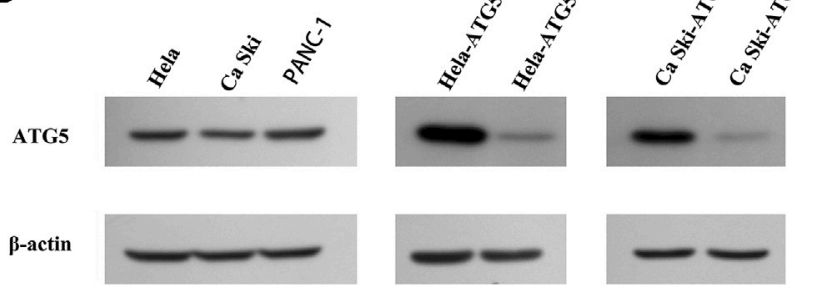

FIGURE 8 | Expression of ATG5 in cervical cancer cell lines (A) The expression of ATG5 mRNA was detected in Hela and Ca Ski (B) After $72 \mathrm{~h}$ infection, Hela and Ca Ski cells with green fluorescence were the cells that transfected plasmid successfully. Magnification $\times 100$ (C) $\mathrm{mRNA}$ expression of ATG5 was significantly inhibited by ATG5-RNAi compared with the control group. Error bars represent \pm SD. ${ }^{\star} p<0.05,{ }^{* \star} p<0.01,{ }^{* \star *} p<0.001$ (D) Protein expression of ATG5 could be detected in Hela and Ca Ski, and was significantly downregulated by ATG5-RNAi in Hela-ATG5-KD and Ca Ski-ATG5-KD cells compared with the control group. PANC-1 was used as a positive control.

and EMT signature-related genes (Choi et al., 2010) such as DPH3, CD24, KRT8, CTNNAL1, EPCAM, TSPAN13, MYB, TPD52, ECHDC1, CLSPN, USP33, TTK, TOM1L1, GCA, COMMD8, CGAS, OSTM1, and EMT score. Tumor tissues had higher EMT scores than normal tissues, and a high EMT score predicted shorter survival time for CESC patients.

\section{Genomic Alterations and Methylation of ARGs in CESC}

Genetic alteration results showed that $16.15 \%$ of patients own mutations of these ARGs. Among these genes, a total of 16 genes have a mutation rate $\geq 1 \%$, of which ATG2B and RB1CC1 are the most frequently mutated genes (4\%). Missense and splice are the two most common types of mutations (Figure 6A). ATG5 was altered in $3(1.03 \%)$ of the 291 CESC patients. Figure 6B shows the mutation type and domain of ATG5. Moreover, concomitant occurrence of ATG5 mutation and ULK2 mutation was found in CESC (Figure 6C). Compared with most mutated ARGs, ATG5 owns a relatively low VAF, except ATG10. MEXPRESS was used for visualizing expression, DNA methylation, and clinical parameters based on TCGA data, and showed the dramatic relationship between ATG5 methylation status and OS in CESC patients (Figure 7A). According to the annotation file of Methylation450 BeadChip platform, there are 19 methylation sites of ATG5, namely, cg24211478, cg14951955, cg00333154, cg07541085, cg15336269, cg07747241, cg01786360, cg16950012, cg22876713, cg10566763, cg26178529, cg01644481, cg02711268, cg14660784, cg18206,736, cg20059537, cg21148531, cg04389950, and cg10850197. As shown in Figure 7B, there was a negative correlation between ATG5 expression and the methylation sites cg02711268, cg18206,736, cg07541085, cg24211478, cg26178529, and cg10850197. The significant sites of those methylation sites in ATG5 on OS in CESC patients were shown in KM including beneficial OS relevance methylation sites such as cg00333154, cg15336269, cg02711268, and cg21148531 and the harmful OSrelated sites such as cg24211478, cg10566763, cg26178529, and cg10850197 (Figures 7C-J).

\section{The Regulation of ATG5 Expression in Cervical Carcinoma Cells}

ATG5 mRNA showed higher expression in cervical cancer cell lines Hela and Ca Ski compared with the cervical epithelial cell line CRL2614 (Figure 8A). The recombinant lentiviral vector containing ATG5-RNAi labeled with green fluorescence was used to downregulate ATG5 expression of Hela and Ca Ski cells. Cells presented with green fluorescence indicated the success of transfection (Figure 8B). RT-qPCR showed that the levels of ATG5 mRNA were significantly downregulated in Hela-KD 

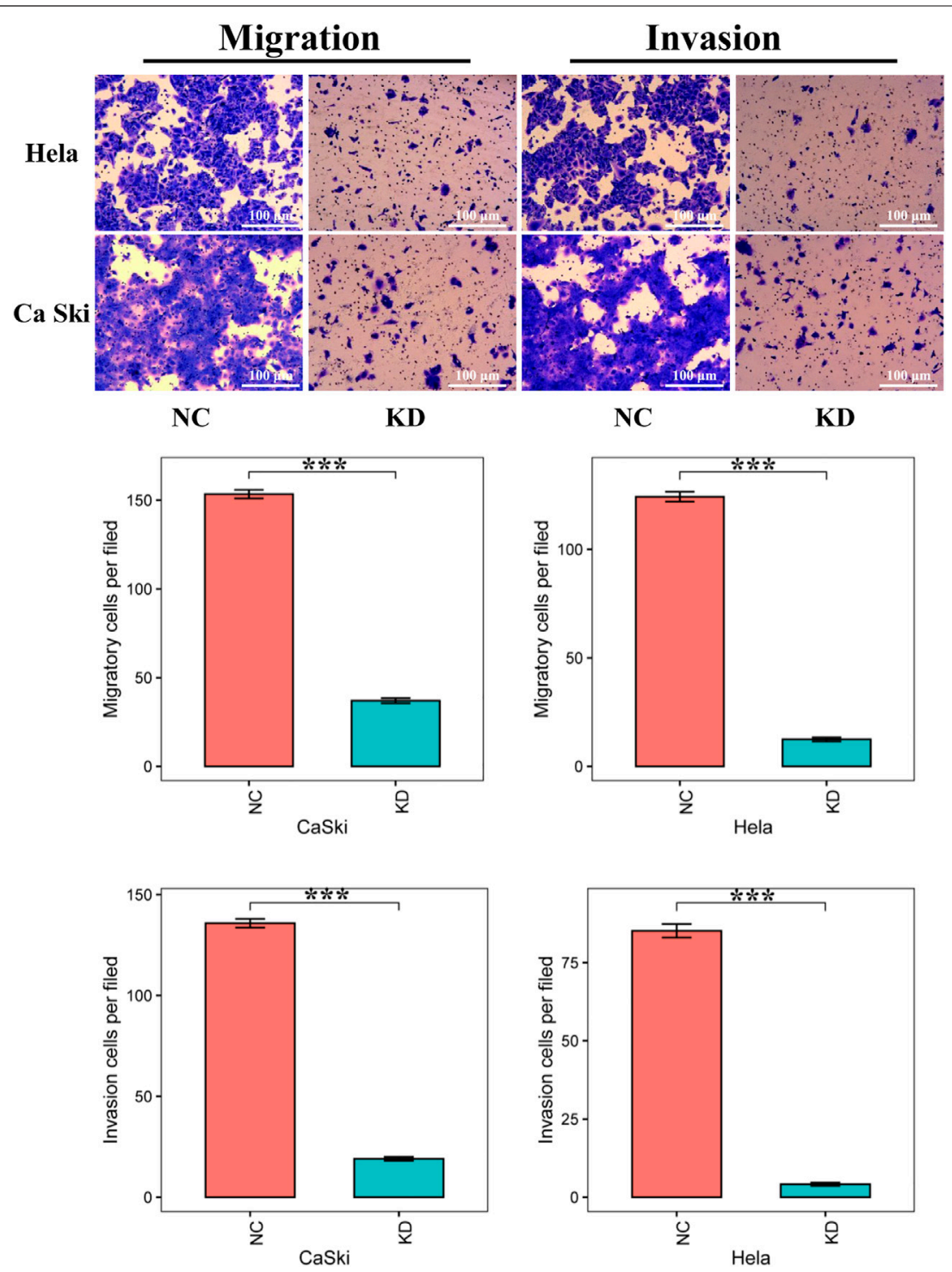

FIGURE 9 | Down-regulation of ATG5 significantly decelerates cervical cancer cells migration and invasion. Magnification $\times 100$. Error bars represent \pm SD. ${ }^{* \star *} p<$ 0.001 .

(knockdown) and Ca Ski-KD cells compared with NC (negative control) cells (Figure 8C, $p<0.01$ ). In addition, the sequence with the highest knockdown efficiency was selected for use in the next study. The knockdown efficiency was further confirmed by Western blot (Figure 8D).

\section{Knockdown of ATG5 Impedes Cervical Cancer Cells Migration and Invasion}

By Transwell migration assay, ATG5 knockdown demonstrated a markedly negative effect on the migratory capacity of Hela and Ca Ski cells compared with NC (Figure 9). Subsequently, the role of ATG5 in invasion was assessed by a Transwell invasion assay. Likewise, the data of the Transwell invasion assay showed that downregulation of ATG5 dramatically suppressed the invasion in cervical cancer cells
(Figure 9). In brief, these results exhibited the repression of ATG5 in the migration and invasion of cervical cancer cells in vitro.

\section{ATG5 Knockdown Reversed EMT of Cervical Cancer Cells}

Further investigation found that knockdown of ATG5 in Hela cells downregulated expressions of interstitial phenotypic markers (N-cadherin and Vimentin), EMT signaling pathwayrelated proteins (P-ERK, P-NFkBp65, and P-mTOR), extracellular matrix metalloproteinases (MMP-9), CC-specific interstitial phenotypic marker (Fibronectin), and EMT-related transcription factors (Twist), but upregulated epithelial marker E-cadherin (Figure 10). However, other markers including NFkBp65, mTOR, P38, AKT, P-P38, P-AKT, MMP-2, 


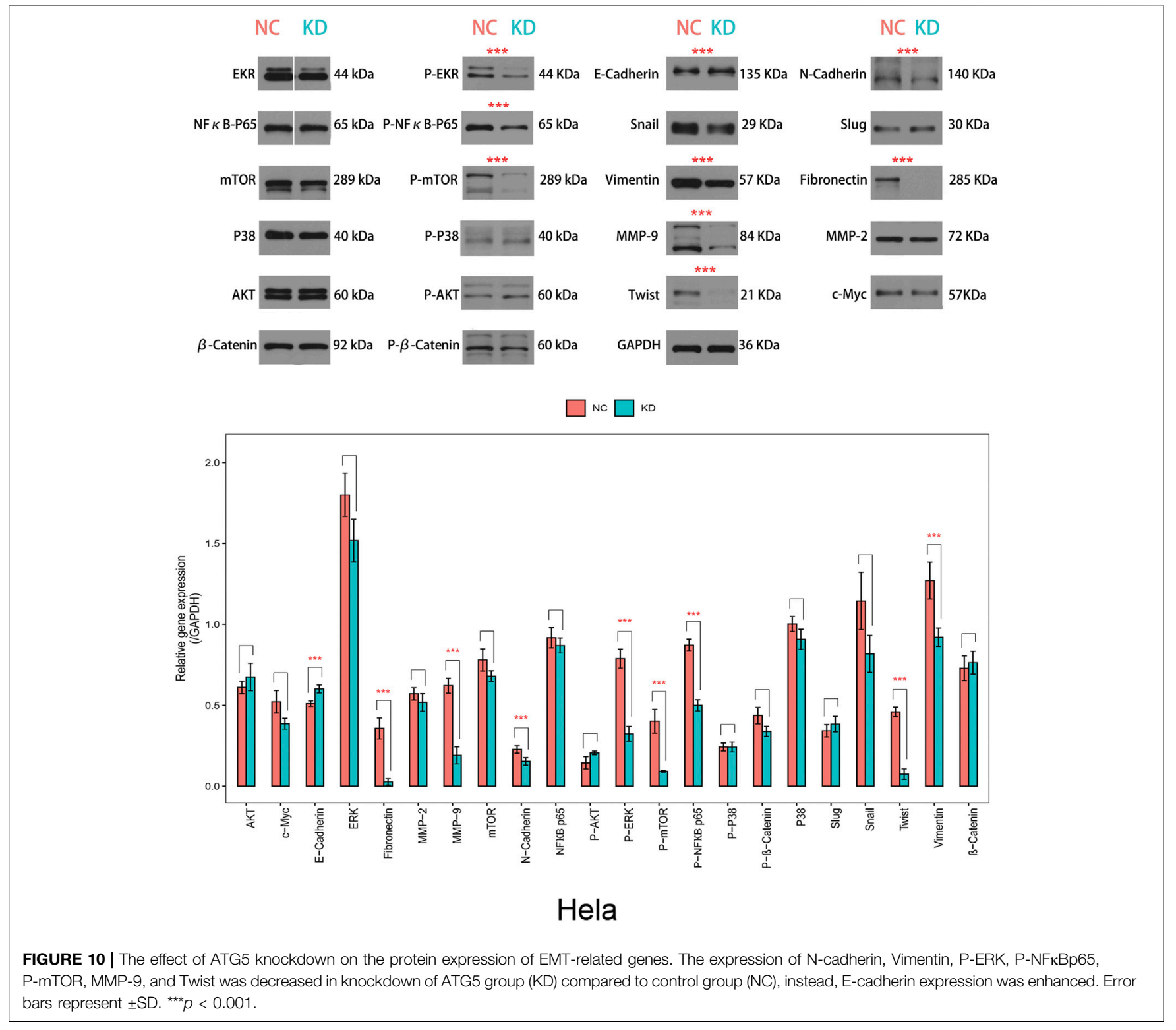

$\beta$-Catenin, Slug, P- $\beta$-Catenin, $c-M y c$, and Snail were not affected by ATG5 knockdown. Generally, downregulation of ATG5 could reverse the EMT process by specific pathways in cervical cancer cells and resulted in attenuation of migration and invasion.

\section{DISCUSSION}

Autophagy is an essential process induced under various conditions of cellular stress and tightly regulated to respond correctly to the ever-changing environment. It is now apparent that autophagy plays different roles in cancer progression. It plays a cancer inhibition role in the onset of tumorigenesis and cancer promotion in the cancer advancement phase (White, 2012). Autophagy inhibition may be an effective anti-cancer therapy in advanced cancer (Barbeau et al., 2020). Invasion and metastasis contribute to a high recurrence rate, which is the main cause of the poor prognosis of cancer patients (Malek et al., 2014). Despite advances in cancer management, some patients with cervical cancer undergoing early metastasis, especially lymph node metastasis, would ultimately lead to poor clinical outcomes (Nanthamongkolkul and Hanprasertpong, 2018; Xu et al., 2018). EMT, defined as a process by which cancer cells change from an epithelial phenotype to a mesenchymal phenotype, promotes invasion and metastasis by enhancing the capability of cancer cells to invade and migrate (Lamouille et al., 2014). Novel increasing evidence showed that the process of EMT could be promoted or inhibited by autophagy (Catalano et al., 2015; Dash et al., 2018; Daskalaki et al., 2018; Wang et al., 2019; Wu et al., 2019; Liu et al., 2020), and the core ARGs involved in the autophagy process plays important roles in EMT regulation (Peng et al., 2013; Liu et al., 2020). 


\section{ATG5 EXPRESSION AND METHYLATION}

The prognosis of cervical cancer

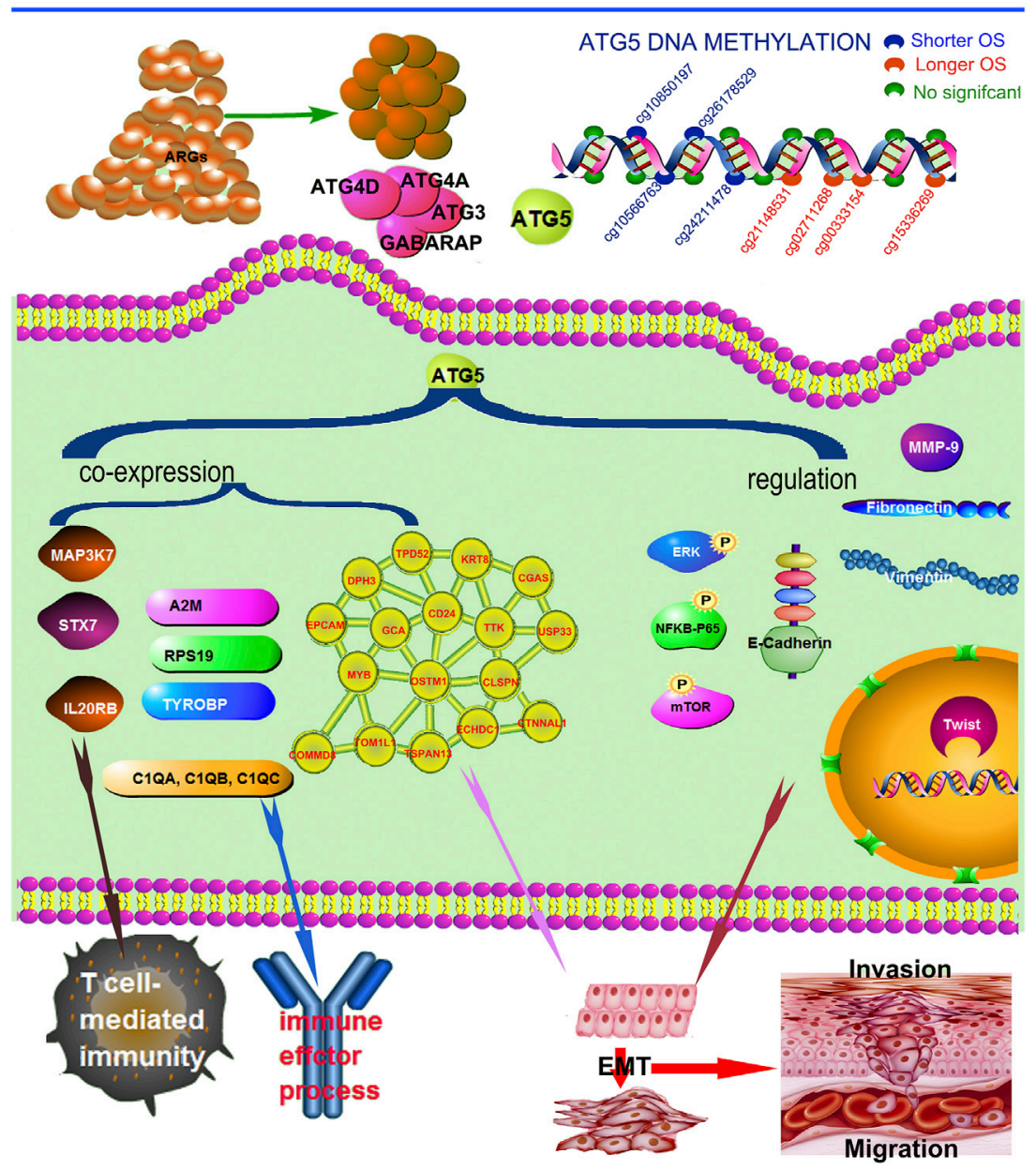

FIGURE 11 | The illumination of the effect of ATG5 expression and methylation in cervical cancer. ATG5 among these 32 ARGs was the most significant harmful factor affecting the prognosis of CESC patients. ATG5 co-expressed genes involved in the regulation of the immune effector process were A2M, C1QA, C1QB, C1QC, RPS19, MAP3K7, TYROBP, STX7, and IL20RB. And, MAP3K7, STX7, and IL20RB were in-volved in the regulation of T cell-mediated immunity. Moreover, ATG5 might promote the migration and invasion of CESC by the regulation of N-cadherin, Vimentin, P-ERK, P-NFKBp65, P-mTOR, MMP-9, and Twist. ATG5 was also significantly correlated with EMT signature related genes including DPH3, CD24, KRT8, CTNNAL1, EPCAM, TSPAN13, MYB, TPD52, ECHDC1, CLSPN, USP33, TTK, TOM1L1, GCA, COMMD8, CGAS, and OSTM1. ATG5 methyltaion status also influence CESC OS. Among these 19 methylation sites in ATG5, four sites significantly increased while other four sites significantly decreased OS of cervical cancer patients.

In this study, we analyzed the prognosis of ARG signature in cervical cancer patients. By univariate and multivariate analysis, ATG5, ATG4D, and ATG4A were distinguished as independent prognostic predictors from the TCGA database. It was vital that ATG5 was the only important harmful marker influencing the prognosis of cervical cancer patients. ATG5 is one of the essential regulators of autophagy. Regardless of stage, grade, and pathological characteristics, cervical cancer patients with high expression of ATG5 had shorter survival. Due to the limited sample size ( 29 cases), there was no statistical significance in the adenocarcinoma group. However, the KM curve of the highATG5 group was significantly lower than that of the low-ATG5 group. Our results suggested that ATG5, as one of ARG signatures, was an essential contributor to the poor prognosis of cervical cancer. The higher expression of ATG5 was also associated with poor clinical outcomes of other cancers (Yang et al., 2017; Cheng et al., 2019). Conversely, positive expression of ATG5 predicts a favorable prognosis in patients with breast cancer and osteosarcoma (Wang et al., 2015; Zhao et al., 2018).

Besides the expression profile of ATG5, the hereditary genetic polymorphisms of ATG5 were also recognized as prognostic predictors of early-stage ESCC patients (Yang et al., 2017). Therefore, we investigate the genomic alterations and methylation of ARGs in CESC. Eight methylation sites of ATG5, namely, four sites with good prognosis and four sites with poor prognosis, were explored to be significantly associated with OS of cervical cancer patients. Four sites with beneficial OS (cg00333154, cg15336269, cg02711268, and cg21148531 located at chr6:106774171:106809238 
length $\quad \approx 35 \quad \mathrm{kbp} \quad 10 \quad$ based on illuminaMethyl450_hg19_GPL16304_TCGAlegacy annotation data) and four sites with harmful OS (cg24211478, cg10566763, cg26178529, and cg10850197 located at chr6:106773226:106773720 length $494 \mathrm{bp}$ ) were located in different positions of transcription regions of ATG5. The relation between DNA methylation and gene expression is importantly subjected to the genomic location of DNA methylation, which also affects the clinicopathological characteristics of cancers (van Vlodrop et al., 2011). Traditionally, cancer research predominantly focused on the prognostic significance and effects of promoter $\mathrm{CpG}$ island hypermethylation located in tumor suppressor genes (Jacinto et al., 2007; Amara et al., 2008; Singh et al., 2020). It is well known that DNA methylation of promoters can silence genes while growing evidence suggests that methylation may also be associated with gene activation (Smith et al., 2020). Moreover, methylation within a gene-body or transcribed region does not block the expression of the affected gene (Salem et al., 2000; Zilberman et al., 2007; Yang et al., 2014). These might explain the different prognostic significance among the eight methylation sites of ATG5. In addition, our analysis about genomic alterations and methylation of ATG5 showed the concomitant occurrence of ATG5 mutation and ULK2 mutation and indicated that methylation sites of ATG5 could also serve as prognosis predictors in cervical cancer patients. As reported by others, the inhibition of autophagy by promoter methylation of ULK2, which leads to downregulation of transcript levels, was essential for cancer growth under the genetic background of ATG5 (Shukla et al., 2014). Moreover, ATG5 was identified as one of the immune-related genes in ESCC (Li et al., 2017). Likewise, our study found that ATG5 was significantly correlated with immune-related genes involved in the immune effector process and regulation of T-cell-mediated immunity. Research has found that ATG5 silencing could abolish the EMT promotion by miR-210-5p in osteosarcoma (Liu et al., 2020). Also, in hepatocellular carcinoma, ATG5 downregulation drastically dampened TGF- $\beta 2$-induced EMT (Dash et al., 2018). By contrast, knockdown of ATG5 increased migration and invasion in glioblastoma cells and RAS-mutated cancer cells by promoting EMT (Catalano et al., 2015; Wang et al., 2019). However, the effect of ATG5 on EMT and the prognosis in cervical cancer is unknown to date. We analyzed the relationship between ATG5 and EMT-related gene signature in cervical cancer. In addition, cervical cancer cell lines were used to investigate the effect of ATG5 on migration and invasion.

ATG5 knockdown could inhibit migration and invasion of cervical cancer cells by reversing EMT. N-cadherin, Vimentin, P-ERK, P-NFkBp65, P-mTOR, MMP-9, Fibronectin, and Twist might be the possible mechanisms involved in ATG5-dependent EMT regulation. However, function acquisition and loss of these genes were needed to be carried out in our further epidemical

\section{REFERENCES}

Abudukadeer, A., Bakry, R., Goebel, G., Mutz-Dehbalaie, I., Widschwendter, A., Bonn, G. K., et al. (2012). Clinical Relevance of CDH1 and CDH13 DNA-Methylation in Serum of Cervical Cancer Patients. Ijms 13, 8353-8363. doi:10.3390/ijms13078353 investigation. MMP-9, as a Matrix metalloproteinase, can destroy the integrity of the basement membrane and extracellular matrix and promote the detachment of tumor cells from the endothelium, which will lead to metastatic process (Voura et al., 2013; Rempe et al., 2016). Fibronectin is one of the ECM components and serves as a mesenchymal marker (Paolillo and Schinelli, 2019). Elevated protein expression of $\mathrm{N}$-cadherin and Vimentin promotes mesenchymal phenotypic transition (Paolillo and Schinelli, 2019). ERK-, NFkBp65-, and mTOR-signaling pathways were the important mechanisms implicated in the regulation of the EMT process (Singh et al., 2018).

\section{CONCLUSION}

In summary, ATG5 involved in the EMT process and immune regulation in cervical cancer could affect the survival of cervical cancer patients by expression and methylation level, proposing that ATG5 may be a potentially powerful therapeutic target for cervical cancer (Figure 11).

\section{DATA AVAILABILITY STATEMENT}

The original contributions presented in the study are included in the article/supplementary material, further inquiries can be directed to the corresponding author.

\section{AUTHOR CONTRIBUTIONS}

SZ and YX designed research; XW, JD, and HY performed experiments; SZ and YX analyzed the data; YX, SZ and XW wrote the manuscript. All authors contributed to writing and critically revising the manuscript. All authors have read and agreed to the published version of the manuscript.

\section{FUNDING}

This study was supported by Program of Taizhou Science and Technology Grant (20ywa04).

\section{SUPPLEMENTARY MATERIAL}

The Supplementary Material for this article can be found online at: https://www.frontiersin.org/articles/10.3389/fcell.2021.757184/ full\#supplementary-material

Agrotis, A., von Chamier, L., Oliver, H., Kiso, K., Singh, T., and Ketteler, R. (2019). Human ATG4 Autophagy Proteases Counteract Attachment of Ubiquitin-like LC3/GABARAP Proteins to Other Cellular Proteins. J. Biol. Chem. 294, 12610-12621. doi:10.1074/jbc.AC119.009977

Amara, K., Trimeche, M., Ziadi, S., Laatiri, A., Hachana, M., and Korbi, S. (2008). Prognostic Significance of Aberrant Promoter Hypermethylation of CpG 
Islands in Patients with Diffuse Large B-Cell Lymphomas. Ann. Oncol. 19, 1774-1786. doi:10.1093/annonc/mdn374

Barbeau, L. M. O., Keulers, T. G. H., and Rouschop, K. M. A. (2020). Tumors Responsive to Autophagy-Inhibition: Identification and Biomarkers. Cancers 12, 2463. doi:10.3390/cancers 12092463

Besteiro, S., Brooks, C. F., Striepen, B., and Dubremetz, J.-F. (2011). Autophagy Protein Atg3 Is Essential for Maintaining Mitochondrial Integrity and for normal Intracellular Development of Toxoplasma Gondii Tachyzoites. Plos Pathog. 7, e1002416. doi:10.1371/journal.ppat.1002416

Birgisdottir, Å. B., Lamark, T., and Johansen, T. (2013). The LIR Motif - Crucial for Selective Autophagy. J. Cel Sci 126, 3237-3247. doi:10.1242/jcs.126128

Catalano, M., D’Alessandro, G., Lepore, F., Corazzari, M., Caldarola, S., Valacca, C., et al. (2015). Autophagy Induction Impairs Migration and Invasion by Reversing EMT in Glioblastoma Cells. Mol. Oncol. 9, 1612-1625. doi:10.1016/j.molonc.2015.04.016

Chen, H., Deng, Q., Wang, W., Tao, H., and Gao, Y. (2020). Identification of an Autophagy-Related Gene Signature for Survival Prediction in Patients with Cervical Cancer. J. Ovarian Res. 13, 131. doi:10.1186/s13048-020-00730-8

Cheng, X., Xu, Q., Zhang, Y., Shen, M., Zhang, S., Mao, F., et al. (2019). miR-34a Inhibits Progression of Neuroblastoma by Targeting Autophagy-Related Gene 5. Eur. J. Pharmacol. 850, 53-63. doi:10.1016/j.ejphar.2019.01.071

Choi, Y.-L., Bocanegra, M., Kwon, M. J., Shin, Y. K., Nam, S. J., Yang, J.-H., et al. (2010). LYN Is a Mediator of Epithelial-Mesenchymal Transition and a Target of Dasatinib in Breast Cancer. Cancer Res. 70, 2296-2306. doi:10.1158/0008-5472.CAN-09-3141

Dash, S., Sarashetti, P. M., Rajashekar, B., Chowdhury, R., and Mukherjee, S. (2018). TGF- $\beta 2$-induced EMT Is Dampened by Inhibition of Autophagy and TNF- $\alpha$ Treatment. Oncotarget 9, 6433-6449. doi:10.18632/oncotarget.23942

Daskalaki, I., Gkikas, I., and Tavernarakis, N. (2018). Hypoxia and Selective Autophagy in Cancer Development and Therapy. Front. Cel Dev. Biol. 6, 104. doi:10.3389/fcell.2018.00104

Diao, J., Liu, R., Rong, Y., Zhao, M., Zhang, J., Lai, Y., et al. (2015). ATG14 Promotes Membrane Tethering and Fusion of Autophagosomes to Endolysosomes. Nature 520, 563-566. doi:10.1038/nature14147

Dikic, I., and Elazar, Z. (2018). Mechanism and Medical Implications of Mammalian Autophagy. Nat. Rev. Mol. Cel Biol 19, 349-364. doi:10.1038/ s41580-018-0003-4

Dooley, H. C., Razi, M., Polson, H. E. J., Girardin, S. E., Wilson, M. I., and Tooze, S. A. (2014). WIPI2 Links LC3 Conjugation with PI3P, Autophagosome Formation, and Pathogen Clearance by Recruiting Atg12-5-16L1. Mol. Cel 55, 238-252. doi:10.1016/j.molcel.2014.05.021

Feng, Y., He, D., Yao, Z., and Klionsky, D. J. (2014). The Machinery of Macroautophagy. Cell Res 24, 24-41. doi:10.1038/cr.2013.168

Hanada, T., and Ohsumi, Y. (2005). Structure-function Relationship of Atg12, a Ubiquitin-like Modifier Essential for Autophagy. Autophagy 1, 110-118. doi:10.4161/auto.1.2.1858

Hänzelmann, S., Castelo, R., and Guinney, J. (2013). GSVA: Gene Set Variation Analysis for Microarray and RNA-Seq Data. BMC Bioinformatics 14, 7. doi:10.1186/1471-2105-14-7

Hu, S., Wang, L., Zhang, X., Wu, Y., Yang, J., and Li, J. (2018). Autophagy Induces Transforming Growth Factor- $\beta$-dependent Epithelial-Mesenchymal Transition in Hepatocarcinoma Cells through cAMP Response Element Binding Signalling. J. Cel Mol Med 22, 5518-5532. doi:10.1111/jcmm.13825

Hurley, J. H., and Young, L. N. (2017). Mechanisms of Autophagy Initiation. Annu. Rev. Biochem. 86, 225-244. doi:10.1146/annurev-biochem-061516-044820

Jacinto, F. V., Ballestar, E., Ropero, S., and Esteller, M. (2007). Discovery of Epigenetically Silenced Genes by Methylated DNA Immunoprecipitation in colon Cancer Cells. Cancer Res. 67, 11481-11486. doi:10.1158/0008-5472.CAN-07-2687

Jiang, Y., and Zhan, H. (2020). Communication between EMT and PD-L1 Signaling: New Insights into Tumor Immune Evasion. Cancer Lett. 468, 72-81. doi:10.1016/j.canlet.2019.10.013

Jung, C. H., Jun, C. B., Ro, S.-H., Kim, Y.-M., Otto, N. M., Cao, J., et al. (2009). ULK-Atg13-FIP200 Complexes Mediate mTOR Signaling to the Autophagy Machinery. MBoC 20, 1992-2003. doi:10.1091/mbc.E08-12-1249

Kim, B.-W., Jin, Y., Kim, J., Kim, J. H., Jung, J., Kang, S., et al. (2018). The C-Terminal Region of ATG101 Bridges ULK1 and PtdIns3K Complex in Autophagy Initiation. Autophagy 14, 2104-2116. doi:10.1080/15548627.2018.1504716

Lamouille, S., Xu, J., and Derynck, R. (2014). Molecular Mechanisms of EpithelialMesenchymal Transition. Nat. Rev. Mol. Cel Biol 15, 178-196. doi:10.1038/nrm3758
Li, Y., Lu, Z., Che, Y., Wang, J., Sun, S., Huang, J., et al. (2017). Immune Signature Profiling Identified Predictive and Prognostic Factors for Esophageal Squamous Cell Carcinoma. Oncoimmunology 6, el356147. doi:10.1080/ 2162402X.2017.1356147

Liu, W., Jiang, D., Gong, F., Huang, Y., Luo, Y., Rong, Y., et al. (2020). miR-210-5p Promotes Epithelial-Mesenchymal Transition by Inhibiting PIK3R5 Thereby Activating Oncogenic Autophagy in Osteosarcoma Cells. Cell Death Dis 11, 93. doi:10.1038/s41419-020-2270-1

Malek, E., Jagannathan, S., and Driscoll, J. J. (2014). Correlation of Long Noncoding RNA Expression with Metastasis, Drug Resistance and Clinical Outcome in Cancer. Oncotarget 5, 8027-8038. doi:10.18632/oncotarget.2469

Matoba, K., Kotani, T., Tsutsumi, A., Tsuji, T., Mori, T., Noshiro, D., et al. (2020). Atg9 Is a Lipid Scramblase that Mediates Autophagosomal Membrane Expansion. Nat. Struct. Mol. Biol. 27, 1185-1193. doi:10.1038/s41594-02000518-w

Mayakonda, A., Lin, D.-C., Assenov, Y., Plass, C., and Koeffler, H. P. (2018). Maftools: Efficient and Comprehensive Analysis of Somatic Variants in Cancer. Genome Res. 28, 1747-1756. doi:10.1101/gr.239244.118

Meir, H., Kenter, G., Burggraaf, J., Kroep, J., Welters, M., Melief, C., et al. (2014). The Need for Improvement of the Treatment of Advanced and Metastatic Cervical Cancer, the Rationale for Combined Chemo-Immunotherapy. Acamc 14, 190-203. doi:10.2174/18715206113136660372

Mesquita, A., Cardenal-Muñoz, E., Dominguez, E., Muñoz-Braceras, S., NuñezCorcuera, B., Phillips, B. A., et al. (2017). Autophagy in Dictyostelium: Mechanisms, Regulation and Disease in a Simple Biomedical Model. Autophagy 13, 24-40. doi:10.1080/15548627.2016.1226737

Mizushima, N., Yoshimori, T., and Ohsumi, Y. (2011). The Role of Atg Proteins in Autophagosome Formation. Annu. Rev. Cel Dev. Biol. 27, 107-132. doi:10.1146/annurev-cellbio-092910-154005

Moore, L. D., Le, T., and Fan, G. (2013). DNA Methylation and its Basic Function. Neuropsychopharmacol 38, 23-38. doi:10.1038/npp.2012.112

Nanthamongkolkul, K., and Hanprasertpong, J. (2018). Predictive Factors of Pelvic Lymph Node Metastasis in Early-Stage Cervical Cancer. Oncol. Res. Treat. 41, 194-198. doi:10.1159/000485840

Nieto, M. A., Huang, R. Y.-J., Jackson, R. A., and Thiery, J. P. (2016). Emt: 2016. Cell 166, 21-45. doi:10.1016/j.cell.2016.06.028

Otomo, T., and Maeda, S. (2019). ATG2A Transfers Lipids between Membranes In Vitro. Autophagy 15, 2031-2032. doi:10.1080/15548627.2019.1659622

Overmeer, R. M., Louwers, J. A., Meijer, C. J. L. M., van Kemenade, F. J., Hesselink, A. T., Daalmeijer, N. F., et al. (2011). Combined CADM1 and MAL Promoter Methylation Analysis to Detect (Pre-)malignant Cervical Lesions in High-Risk HPV-Positive Women. Int. J. Cancer 129, 2218-2225. doi:10.1002/ijc.25890

Paolillo, M., and Schinelli, S. (2019). Extracellular Matrix Alterations in Metastatic Processes. Ijms 20, 4947. doi:10.3390/ijms20194947

Peng, Y.-F., Shi, Y.-H., Ding, Z.-B., Ke, A.-W., Gu, C.-Y., Hui, B., et al. (2013). Autophagy Inhibition Suppresses Pulmonary Metastasis of HCC in Mice via Impairing Anoikis Resistance and Colonization of HCC Cells. Autophagy 9, 2056-2068. doi:10.4161/auto.26398

Phillips, A. R., Suttangkakul, A., and Vierstra, R. D. (2008). The ATG12-Conjugating Enzyme ATG10 Is Essential for Autophagic Vesicle Formation inArabidopsis Thaliana. Genetics 178, 1339-1353. doi:10.1534/genetics.107.086199

Price, E. M., Cotton, A. M., Lam, L. L., Farré, P., Emberly, E., Brown, C. J., et al. (2013). Additional Annotation Enhances Potential for Biologically-Relevant Analysis of the Illumina Infinium HumanMethylation450 BeadChip Array. Epigenetics \& Chromatin 6, 4. doi:10.1186/1756-8935-6-4

Proikas-Cezanne, T., Takacs, Z., Dönnes, P., and Kohlbacher, O. (2015). WIPI Proteins: Essential PtdIns3P Effectors at the Nascent Autophagosome. J. Cel Sci 128, 207-217. doi:10.1242/jcs. 146258

Rempe, R. G., Hartz, A. M., and Bauer, B. (2016). Matrix Metalloproteinases in the Brain and Blood-Brain Barrier: Versatile Breakers and Makers. J. Cereb. Blood Flow Metab. 36, 1481-1507. doi:10.1177/0271678X16655551

Salem, C. E., Markl, I. D. C., Bender, C. M., Gonzales, F. A., Jones, P. A., and Liang, G. (2000). PAX6 Methylation and Ectopic Expression in Human Tumor Cells. Int. J. Cancer 87, 179-185. doi:10.1002/1097-0215(20000715)87:2<179:aidijc4 $>3.0 . c 0 ; 2-\mathrm{x}$

Shi, H., Zhong, F., Yi, X., Shi, Z., Ou, F., Xu, Z., et al. (2020). Application of an Autophagy-Related Gene Prognostic Risk Model Based on TCGA Database in Cervical Cancer. Front. Genet. 11, 616998. doi:10.3389/fgene.2020.616998 
Shukla, S., Patric, I. R. P., Patil, V., Shwetha, S. D., Hegde, A. S., Chandramouli, B. A., et al. (2014). Methylation Silencing of ULK2, an Autophagy Gene, Is Essential for Astrocyte Transformation and Tumor Growth. J. Biol. Chem. 289, 22306-22318. doi:10.1074/jbc.M114.567032

Singh, M., Yelle, N., Venugopal, C., and Singh, S. K. (2018). EMT: Mechanisms and Therapeutic Implications. Pharmacol. Ther. 182, 80-94. doi:10.1016/ j.pharmthera.2017.08.009

Singh, N., Rashid, S., Rashid, S., Dash, N. R., Gupta, S., and Saraya, A. (2020). Clinical Significance of Promoter Methylation Status of Tumor Suppressor Genes in Circulating DNA of Pancreatic Cancer Patients. J. Cancer Res. Clin. Oncol. 146, 897-907. doi:10.1007/s00432-020-03169-y

Small, W., Jr., Bacon, M. A., Bajaj, A., Chuang, L. T., Fisher, B. J., Harkenrider, M. M., et al. (2017). Cervical Cancer: A Global Health Crisis. Cancer 123, 2404-2412. doi:10.1002/cncr.30667

Smith, J., Sen, S., Weeks, R. J., Eccles, M. R., and Chatterjee, A. (2020). Promoter DNA Hypermethylation and Paradoxical Gene Activation. Trends Cancer 6, 392-406. doi:10.1016/j.trecan.2020.02.007

Sun, Y., Li, S., Shen, K., Ye, S., Cao, D., and Yang, J. (2015). DAPK1, MGMT and RARB Promoter Methylation as Biomarkers for High-Grade Cervical Lesions. Int. J. Clin. Exp. Pathol. 8, 14939-14945.

Sung, H., Ferlay, J., Siegel, R. L., Laversanne, M., Soerjomataram, I., Jemal, A., et al. (2021). Global Cancer Statistics 2020: GLOBOCAN Estimates of Incidence and Mortality Worldwide for 36 Cancers in 185 Countries. CA A. Cancer J. Clin. 71, 209-249. doi:10.3322/caac.21660

Tang, Z., Takahashi, Y., Chen, C., Liu, Y., He, H., Tsotakos, N., et al. (2017). Atg2A/ B Deficiency Switches Cytoprotective Autophagy to Non-canonical Caspase-8 Activation and Apoptosis. Cell Death Differ 24, 2127-2138. doi:10.1038/ cdd.2017.133

Tanida, I., Yamasaki, M., Komatsu, M., and Ueno, T. (2012). The FAP Motif within Human ATG7, an Autophagy-Related E1-like Enzyme, Is Essential for the E2Substrate Reaction of LC3 Lipidation. Autophagy 8, 88-97. doi:10.4161/ auto.8.1.18339

van Vlodrop, I. J. H., Niessen, H. E. C., Derks, S., Baldewijns, M. M. L. L., van Criekinge, W., Herman, J. G., et al. (2011). Analysis of Promoter CpG Island Hypermethylation in Cancer: Location, Location, Location!: Figure 1. Clin. Cancer Res. 17, 4225-4231. doi:10.1158/1078-0432.CCR-10-3394

Vij, A., Randhawa, R., Parkash, J., and Changotra, H. (2016). Investigating Regulatory Signatures of Human Autophagy Related Gene 5 (ATG5) through Functional In Silico Analysis. Meta Gene 9, 237-248. doi:10.1016/ j.mgene.2016.07.012

Voura, E. B., English, J. L., Yu, H.-Y. E., Ho, A. T., Subarsky, P., Hill, R. P., et al. (2013). Proteolysis during Tumor Cell Extravasation In Vitro: Metalloproteinase Involvement across Tumor Cell Types. PLoS One 8, e78413. doi:10.1371/journal.pone.0078413

Wang, L., Yao, L., Zheng, Y.-Z., Xu, Q., Liu, X.-P., Hu, X., et al. (2015). Expression of Autophagy-Related Proteins ATG5 and FIP200 Predicts Favorable Diseasefree Survival in Patients with Breast Cancer. Biochem. Biophysical Res. Commun. 458, 816-822. doi:10.1016/j.bbrc.2015.02.037

Wang, X.-q., Xu, S.-w., Wang, W., Piao, S.-z., Mao, X.-l., Zhou, X.-b., et al. (2021). Identification and Validation of a Novel DNA Damage and DNA Repair Related Genes Based Signature for Colon Cancer Prognosis. Front. Genet. 12, 635863. doi:10.3389/fgene.2021.635863

Wang, Y., Sun, L., Qiu, W., Qi, W., Qi, Y., Liu, Z., et al. (2020). Inhibiting Forkhead Box K1 Induces Autophagy to Reverse Epithelial-Mesenchymal Transition and Metastasis in Gastric Cancer by Regulating Myc-Associated Zinc finger Protein in an Acidic Microenvironment. Aging 12, 6129-6150. doi:10.18632/aging.103013

Wang, Y., Xiong, H., Liu, D., Hill, C., Ertay, A., Li, J., et al. (2019). Autophagy Inhibition Specifically Promotes Epithelial-Mesenchymal Transition and Invasion in RAS-Mutated Cancer Cells. Autophagy 15, 886-899. doi:10.1080/15548627.2019.1569912

Wei, T.-Y, and Simko, V. (2021). R Package 'corrplot': Visualization of a Correlation Matrix. Version 0.90. CRAN. Available at https://github.com/taiyun/corrplot.

Wentzensen, N., Sherman, M. E., Schiffman, M., and Wang, S. S. (2009). Utility of Methylation Markers in Cervical Cancer Early Detection: Appraisal of the State-Of-The-Science. Gynecol. Oncol. 112, 293-299. doi:10.1016/ j.ygyno.2008.10.012

White, E. (2012). Deconvoluting the Context-dependent Role for Autophagy in Cancer. Nat. Rev. Cancer 12, 401-410. doi:10.1038/nrc3262
Wu, H., Lu, X.-X., Wang, J.-R., Yang, T.-Y., Li, X.-M., He, X.-S., et al. (2019). TRAF6 Inhibits Colorectal Cancer Metastasis through Regulating Selective Autophagic CTNNB1/ $\beta$-Catenin Degradation and Is Targeted for GSK3B/ GSK3 $\beta$-Mediated Phosphorylation and Degradation. Autophagy 15, 1506-1522. doi:10.1080/15548627.2019.1586250

Xiong, Q., Li, W., Li, P., Yang, M., Wu, C., and Eichinger, L. (2018). The Role of ATG16 in Autophagy and the Ubiquitin Proteasome System. Cells 8, 2. doi: $10.3390 /$ cells 8010002

Xu, F., Li, Y., Fan, L., Ma, J., Yu, L., Yi, H., et al. (2018). Preoperative SCC-Ag and Thrombocytosis as Predictive Markers for Pelvic Lymphatic Metastasis of Squamous Cervical Cancer in Early FIGO Stage. J. Cancer 9, 1660-1666. doi:10.7150/jca.24049

Yamaguchi, M., Noda, N. N., Nakatogawa, H., Kumeta, H., Ohsumi, Y., and Inagaki, F. (2010). Autophagy-related Protein 8 (Atg8) Family Interacting Motif in Atg3 Mediates the Atg3-Atg8 Interaction and Is Crucial for the Cytoplasm-To-Vacuole Targeting Pathway. J. Biol. Chem. 285, 29599-29607. doi:10.1074/jbc.M110.113670

Yang, M.-C., Wang, H.-C., Hou, Y.-C., Tung, H.-L., Chiu, T.-J., and Shan, Y.-S. (2015). Blockade of Autophagy Reduces Pancreatic Cancer Stem Cell Activity and Potentiates the Tumoricidal Effect of Gemcitabine. Mol. Cancer 14, 179. doi:10.1186/s12943-015-0449-3

Yang, P.-W., Hsieh, M.-S., Chang, Y.-H., Huang, P.-M., and Lee, J.-M. (2017). Genetic Polymorphisms of ATG5 Predict Survival and Recurrence in Patients with Early-Stage Esophageal Squamous Cell Carcinoma. Oncotarget 8, 91494-91504. doi:10.18632/oncotarget.20793

Yang, X., Han, H., De Carvalho, D. D., Lay, F. D., Jones, P. A., and Liang, G. (2014). Gene Body Methylation Can Alter Gene Expression and Is a Therapeutic Target in Cancer. Cancer Cell 26, 577-590. doi:10.1016/j.ccr.2014.07.028

Zachari, M., and Ganley, I. G. (2017). The Mammalian ULK1 Complex and Autophagy Initiation. Essays Biochem. 61, 585-596. doi:10.1042/EBC20170021

Zada, S., Hwang, J. S., Ahmed, M., Lai, T. H., Pham, T. M., Elashkar, O., et al. (2021). Cross Talk between Autophagy and Oncogenic Signaling Pathways and Implications for Cancer Therapy. Biochim. Biophys. Acta (Bba) - Rev. Cancer 1876, 188565. doi:10.1016/j.bbcan.2021.188565

Zhang, L., Li, J., Ouyang, L., Liu, B., and Cheng, Y. (2016). Unraveling the Roles of Atg4 Proteases from Autophagy Modulation to Targeted Cancer Therapy. Cancer Lett. 373, 19-26. doi:10.1016/j.canlet.2016.01.022

Zhao, G.-s., Gao, Z.-r., Zhang, Q., Tang, X.-f., Lv, Y.-f., Zhang, Z.-s., et al. (2018). TSSC3 Promotes Autophagy via Inactivating the Src-Mediated PI3K/Akt/mTOR Pathway to Suppress Tumorigenesis and Metastasis in Osteosarcoma, and Predicts a Favorable Prognosis. J. Exp. Clin. Cancer Res. 37, 188. doi:10.1186/s13046-018-0856-6

Zhao, Y., Zou, Z., Sun, D., Li, Y., Sinha, S. C., Yu, L., et al. (2020). GLIPR2 Is a Negative Regulator of Autophagy and the BECN1-ATG14-Containing Phosphatidylinositol 3-kinase Complex. Autophagy 17, 2891-2904. doi:10.1080/15548627.2020.1847798

Zhou, Y., Zhou, B., Pache, L., Chang, M., Khodabakhshi, A. H., Tanaseichuk, O., et al. (2019). Metascape Provides a Biologist-Oriented Resource for the Analysis of Systems-Level Datasets. Nat. Commun. 10, 1523. doi:10.1038/s41467-019-09234-6

Zilberman, D., Gehring, M., Tran, R. K., Ballinger, T., and Henikoff, S. (2007). Genome-wide Analysis of Arabidopsis thaliana DNA Methylation Uncovers an Interdependence between Methylation and Transcription. Nat. Genet. 39, 61-69. doi:10.1038/ng1929

Conflict of Interest: The authors declare that the research was conducted in the absence of any commercial or financial relationships that could be construed as a potential conflict of interest.

Publisher's Note: All claims expressed in this article are solely those of the authors and do not necessarily represent those of their affiliated organizations, or those of the publisher, the editors and the reviewers. Any product that may be evaluated in this article, or claim that may be made by its manufacturer, is not guaranteed or endorsed by the publisher.

Copyright $\odot 2021$ Zhou, Wang, Ding, Yang and Xie. This is an open-access article distributed under the terms of the Creative Commons Attribution License (CC BY). The use, distribution or reproduction in other forums is permitted, provided the original author(s) and the copyright owner(s) are credited and that the original publication in this journal is cited, in accordance with accepted academic practice. No use, distribution or reproduction is permitted which does not comply with these terms. 